\title{
TITLE:
}

\section{High-spin electronic interaction of small lithium and sodium cluster formation in the excited states}

$\operatorname{AUTHOR}(\mathrm{S}):$

Hotta, S; Doi, K; Nakamura, K; Tachibana, A

\section{CITATION:}

Hotta, S ... [et al]. High-spin electronic interaction of small lithium and sodium cluster formation in the excited states. JOURNAL OF CHEMICAL PHYSICS 2002, 117(1): 142-152

\section{ISSUE DATE:}

2002-07-01

URL:

http://hdl.handle.net/2433/39739

\section{RIGHT:}

Copyright 2002 American Institute of Physics. This article may be downloaded for personal use only. Any other use requires prior permission of the author and the American Institute of Physics. 


\title{
High-spin electronic interaction of small lithium and sodium cluster formation in the excited states
}

\author{
Shigeru Hotta, Kentaro Doi, Koichi Nakamura, and Akitomo Tachibana ${ }^{\text {a) }}$ \\ Department of Engineering Physics and Mechanics, Kyoto University, Kyoto 606-8501, Japan
}

(Received 14 December 2001; accepted 2 April 2002)

\begin{abstract}
We have carried out the MRCI $a b$ initio calculations for small lithium and sodium clusters, and elucidate the interaction between atoms in various high-spin electronic states, in terms of the quantum mechanical energy densities based on the regional density functional theory [Tachibana, J. Chem. Phys. 115, 3497 (2001)]. When the separated two electronic drop regions, where the electronic kinetic-energy density is positive, connect to each other, it is observed that ratios of occupation on configurations change rapidly in the $\mathrm{Li}_{2}$ molecule. These results are considered as one of the evidences that valence electrons can move around both two $\mathrm{Li}$ atoms freely in the meaning of classical mechanics. The shape of electronic drop region depends strongly on the electronic state and represents the characteristics of interaction clearly, and the electronic tension density also gives new images of microscopic electronic stresses. Furthermore, we have clarified the most stable structures of $\mathrm{Li}_{3}$ and $\mathrm{Li}_{4}$ for the high-spin electronic state, which are respectively different from the most stable structures for the low-spin electronic state. The stabilization energy due to taking in a $\mathrm{Li}$ atom is raised gradually as the number of atoms in $\mathrm{Li}_{n}$ cluster increases in the initial stage of cluster propagation. The formation energies of $\mathrm{Na}_{2}, \mathrm{Na}_{3}$, and $\mathrm{Na}_{4}$ clusters are much smaller than that of the corresponding lithium clusters. (c) 2002 American Institute of Physics.
\end{abstract}

[DOI: $10.1063 / 1.1480869$ ]

\section{INTRODUCTION}

One of the most attractive fields of recent experimental study on quantum mechanics is achievement of the BoseEinstein condensation (BEC) ${ }^{1-3}$ The BEC enables us to observe quantum-mechanical phenomena at macroscopic scale: for examples, superfluidity of ${ }^{4} \mathrm{He}$ and superconductivity due to the Cooper pair of electrons. However, the BEC of hydrogen atoms and alkali metal atoms, which have only a small interaction with each other, had not been observed for a long time. Recently, by an advance of experimental techniques, the BECs of alkali atoms were observed successively such as ${ }^{87} \mathrm{Rb},{ }^{4}{ }^{23} \mathrm{Na},{ }^{5}$ and ${ }^{7} \mathrm{Li}^{6,7}$ in 1995 , and at length, the BEC of hydrogen atoms ${ }^{8}$ were achieved in 1998. Interference fringes by the matter wave can be observed in these BECs, ${ }^{9}$ and the applications to atomic laser is expected.

Generally, the BEC is achieved by particles with repulsive interaction because the fluctuation of density is suppressed. On the other hand, it had been conjectured that particles with attractive interaction could not bring about the BEC because the formation of high-density region caused instability. Actually, ${ }^{87} \mathrm{Rb},{ }^{23} \mathrm{Na},{ }^{1} \mathrm{H},{ }^{4} \mathrm{He}$, and the Cooper pair of electrons have repulsive interaction and form stable BEC. However, it is observed that ${ }^{7} \mathrm{Li}$ forms BEC although it has attractive interaction in the usual gas state. ${ }^{6}$ In the the observation of the BEC of ${ }^{7} \mathrm{Li}$, a cold $\mathrm{Li}$ atom is caged up by the magnetic trap which is produced by six permanent magnet cylinders, ${ }^{10}$ and then, the caged atoms feel repulsive

\footnotetext{
a) Author to whom correspondence should be addressed. Electronic mail: akitomo@scl.kyoto-u.ac.jp
}

force effectively, which is originated in the uncertainly principle between the position and the momentum. It is considered that the $\mathrm{BEC}$ of ${ }^{7} \mathrm{Li}$ is a metastable state standing on a subtle balance of the repulsive energy originated in the uncertainly principle, the energy due to the magnetic trap, and the energy due to the attractive interaction between atoms.

In order to understand the interaction among ${ }^{7} \mathrm{Li}$ atoms in the BEC, we need a detailed analysis of the interaction among Li atoms in the small Li cluster system. In particular, the high-spin electronic states of small Li clusters are necessarily important because the $\mathrm{BEC}$ of ${ }^{7} \mathrm{Li}$ occurs under the magnetic trap. ${ }^{10}$ Fortunately, a lot of experimental and theoretical studies on potential-energy curves for various electronic states of $\mathrm{Li}_{2}$ molecule have been reported. ${ }^{11-21}$ Recent progress in technique, to assemble ultracold atoms, has stimulated the construction of schemes that may lead to the production of translationally ultracold molecules in specific rotational and vibrational levels. ${ }^{22-27}$ In particular, the most noteworthy point is that the lowest excited state of $\mathrm{Li}_{2}, a$ ${ }^{3} \Sigma_{u}^{+}$, is very slightly bound, having only 11 vibrational levels. ${ }^{28-30}$ The minimum of the potential-energy curve for the ${ }^{3} \Sigma_{u}^{+}$state can be simulated by the multiconfiguration self-consistent field (MCSCF) method or the superiors. ${ }^{11-21}$ In other words, the potential-energy curve for the ${ }^{3} \Sigma_{u}^{+}$state is monotonically repulsive by the SCF calculations with a single Slater determinant. Therefore, the consideration to occupation on other electronic configurations within conserving the ${ }^{3} \Sigma_{u}^{+}$symmetry should play an important role in the change of potential-energy curve from a repulsive one to an attractive one. 

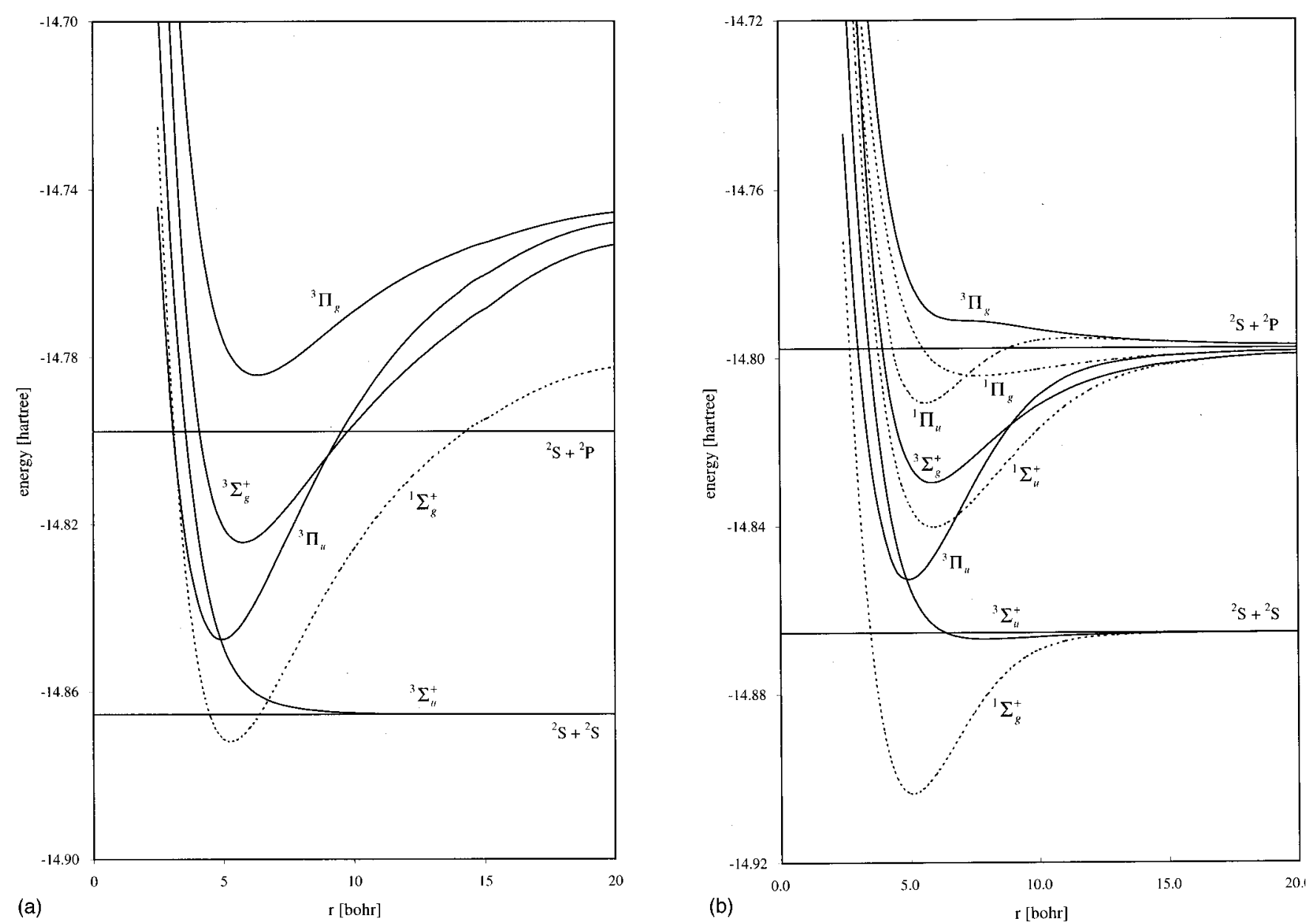

FIG. 1. Potential-energy curves for each electronic state of $\mathrm{Li}_{2}$ molecule at (a) the RHF, (b) the MCSCF, and (c) the MRCI levels. Curves by solid line and dashed line, respectively denote the triplet states and the singlet states.

In addition, a lot of research groups have reported structures of $\mathrm{Li}_{n}$ cluster. $^{31-38}$ Information of geometry is very important for the mechanism of propagation of Li cluster. However, the most stable geometries of $\mathrm{Li}_{n}$ cluster for the highspin electronic state have hardly been reported, unexpectedly. In this paper, we shall elucidate the interaction among $\mathrm{Li}$ atoms in the $\mathrm{Li}_{2}$ and $\mathrm{Li}_{3}$ molecule system in the high-spin electronic states in terms of the regional density functional theory ${ }^{38-49}$ by using high-qualitative $a b$ initio electronic-state calculations. We have treated the triplet states of $\mathrm{Li}_{2}$ molecule, ${ }^{3} \Sigma_{u}^{+},{ }^{3} \Pi_{u},{ }^{3} \Sigma_{g}^{+}$, and ${ }^{3} \Pi_{g}$ states, and the quartet states of $\mathrm{Li}_{3}$ cluster system. Furthermore, calculations of the quintet states of $\mathrm{Li}_{4}$ clusters have been also performed, and the propagation of $\mathrm{Li}_{n}$ cluster for the highspin electronic state has been discussed. We have applied the quantum-mechanical energy densities ${ }^{43}$ based on the regional density functional theory, and have shown the local electronic nature in the propagation of lithium clusters for various electronic states. We have also calculated $\mathrm{Na}_{n}$ clusters $(n=2-4)$ for the high-spin electronic state, and distinguishing characteristics of interaction in lithium clusters from other alkali metals have been discussed by comparing between lithium and sodium clusters.

\section{COMPUTATIONAL METHODS OF ELECTRONIC-STATE CALCULATIONS}

\section{A. Electronic-state calculations of lithium clusters}

$A b$ initio molecular computations reported in this paper were performed with Molecular Regional DFT program package. $^{50}$ The Roos extended Gaussian functions $(6 s 5 p 3 d 2 f)^{51}$ were adopted as the basis set for electronicstate calculations of $\mathrm{Li}_{2}$ molecules. We investigated the potential-energy curves of low-lying eight electronic states by the restricted Hartree-Fock (RHF) level, the MCSCF level, and the internally contracted multireference configuration interaction (MRCI) calculations, ${ }^{52,53}$ respectively. For $\mathrm{Li}_{2}$ molecule, the active space of the MCSCF calculations is composed of $21\left(2-5 \sigma_{g}, 2-4 \sigma_{u}^{*}, 1-3 \pi_{u}, 1-2 \pi_{g}^{*}, 1 \delta_{g}\right.$, and $1 \delta_{u}^{*}$ ) molecular orbitals (MOs) for two valence electrons. The MRCI calculations were carried out by taking into account internal excitations, and singly and doubly external excitations from the core orbitals $\left(1 \sigma_{g}\right.$ and $\left.1 \sigma_{u}^{*}\right)$ and the active space of the MCSCF method.

For electronic-state calculations of $\mathrm{Li}_{3}$ clusters, we adopted the $6-31 \mathrm{G}^{*}$ basis set. ${ }^{54-56}$ We have investigated the colinear structure ${ }^{4} \Sigma^{+}$and the triangle structure ${ }^{4} B_{2}$ in the 


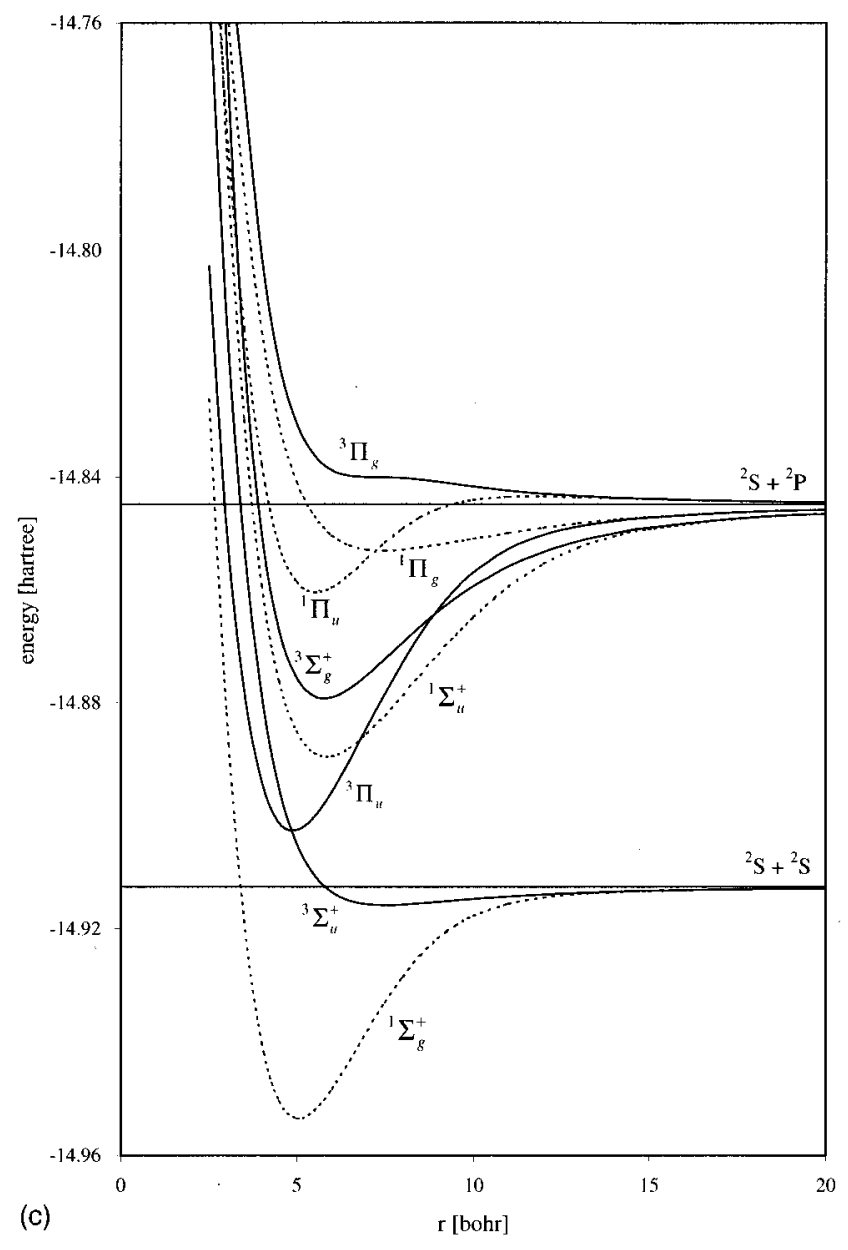

FIG. 1. (Continued.)

quartet state. Both electronic states can be formed by approaching $\mathrm{Li}$ atom of the ${ }^{2} S$ state to $\mathrm{Li}_{2}$ molecule of the ${ }^{3} \Sigma_{u}^{+}$ state with parallel spins. Ab initio calculations at the RHF level, the MCSCF level, and the MRCI level were performed. The active space of the MCSCF calculations is composed of $12 \mathrm{MOs}$, which are originated in $2 s$ and $2 p$ atomic orbitals of each $\mathrm{Li}$ atom, for three valence electrons. The electronic state of the triangle structure ${ }^{4} A_{2}$ has been also calculated. Furthermore, we have calculated some of $\mathrm{Li}_{4}$ clusters with the $6-31 \mathrm{G}^{*}$ basis set with the same quality to the calculations of $\mathrm{Li}_{3}$ clusters, that is, the active space of the MCSCF calculations is composed of 16 MOs for four valence electrons.

\section{B. Electronic-state calculations of sodium clusters}

We adopted the Roos extended Gaussian functions $(7 s 6 p 4 d 3 f)^{51}$ as the basis set for electronic-state calculations of $\mathrm{Na}_{2}$ molecules. For the calculation at the MCSCF level, the active space is composed of $13\left(4-5 \sigma_{g}, 4 \sigma_{u}^{*}\right.$, $2-3 \pi_{u}, 2 \pi_{g}^{*}, 1 \delta_{g}$, and $\left.1 \delta_{u}^{*}\right)$ MOs for two valence electrons. The MRCI calculations were carried out by taking into account internal excitations, and singly and doubly external excitations from the active space of the MCSCF method. Moreover, we have carried out the MCSCF and MRCI calculations of $\mathrm{Na}_{3}$ and $\mathrm{Na}_{4}$ clusters by using a $6-31 \mathrm{G}^{*}$ basis set. The active space of the MCSCF calculations is com-
TABLE I. Optimized length and bond formation energy of $\mathrm{Li}_{2}$ at the MRCI level with Roos' extended Gaussian basis set.

\begin{tabular}{|c|c|c|c|c|}
\hline \multirow[b]{2}{*}{ State } & \multirow[b]{2}{*}{$\begin{array}{l}\text { Optimized } \\
\text { length (bohr) }\end{array}$} & \multirow{2}{*}{$\begin{array}{c}\text { Bond } \\
\text { formation } \\
\text { energy }(e V)\end{array}$} & \multicolumn{2}{|c|}{ Absolute energy (Hartree) } \\
\hline & & & $\begin{array}{l}\text { in optimized } \\
\text { length }\end{array}$ & $\begin{array}{l}\text { in dissociation } \\
\text { limit }\end{array}$ \\
\hline$z_{g}$ & 5.055 & 1.115 & -14.95351 & $-14.91255\left({ }^{2} S+{ }^{2} S\right)$ \\
\hline${ }^{3} \Sigma_{u}^{+}$ & 7.495 & 0.088 & -14.91580 & $-14.91255\left({ }^{2} S+{ }^{2} S\right)$ \\
\hline${ }^{3} \Pi_{u}^{u}$ & 4.887 & 1.573 & -14.90262 & $-14.84480\left({ }^{2} S+{ }^{2} P\right)$ \\
\hline${ }^{1} \Sigma_{u}^{+}$ & 5.862 & 1.217 & -14.88953 & $-14.84480\left({ }^{2} S+{ }^{2} P\right)$ \\
\hline${ }^{3} \Sigma_{g}^{+}$ & 5.787 & 0.936 & -14.87919 & $-14.84480\left({ }^{2} S+{ }^{2} P\right)$ \\
\hline${ }^{1} \Pi_{u}^{8}$ & 5.541 & 0.425 & -14.86042 & $-14.84480\left({ }^{2} S+{ }^{2} P\right)$ \\
\hline${ }^{1} \Pi_{g}^{u}$ & 7.452 & 0.224 & -14.85305 & $-14.84480\left({ }^{2} S+{ }^{2} P\right)$ \\
\hline${ }^{3} \Pi_{g}^{8}$ & $\cdots$ & $\cdots$ & $\cdots$ & $-14.84480\left({ }^{2} S+{ }^{2} P\right)$ \\
\hline
\end{tabular}

posed of 12 and $16 \mathrm{MOs}$ for $\mathrm{Na}_{3}$ and $\mathrm{Na}_{4}$, respectively, which are originated in $3 s$ and $3 p$ atomic orbitals of each $\mathrm{Na}$ atom, for three $\left(\mathrm{Na}_{3}\right)$ or four $\left(\mathrm{Na}_{4}\right)$ valence electrons.

\section{RESULTS AND DISCUSSION}

\section{A. Triplet states of $\mathrm{Li}_{2}$ molecules}

Figure 1 shows the potential-energy curves for each electronic state of $\mathrm{Li}_{2}$ molecule at the RHF, the MCSCF, and the MRCI levels. Shapes and relative positions of each potential curve for the MCSCF and the MRCI calculations are consistent with those in references. ${ }^{11-15}$ We found that the absolute potential energies obtained by the MRCI calculation, without excitations from the core orbitals, almost coincides with those of the MCSCF level. It means that the extent of active space is adequate and that consideration of excitations from the core orbitals contributes strikingly to the absolute potential energy. The optimized $\mathrm{Li}-\mathrm{Li}$ length and the bond formation energy at the MRCI level are listed in Table I. In this paper, we have demonstrated the interaction between two $\mathrm{Li}$ atoms in terms of the quantum-mechanical densities $^{43}$ based on the regional density functional theory. ${ }^{39-49}$ The electronic kinetic-energy density $n_{T}(\vec{r})$ is defined as ${ }^{43}$

$$
\begin{aligned}
n_{T}(\vec{r})= & \frac{1}{2} \sum_{i} \nu_{i}\left(\left\{-\frac{\hbar^{2}}{2 m} \Delta \psi_{i}^{*}(\vec{r})\right\} \psi_{i}(\vec{r})\right. \\
& \left.+\psi_{i}^{*}(\vec{r})\left\{-\frac{\hbar^{2}}{2 m} \Delta \psi_{i}(\vec{r})\right\}\right),
\end{aligned}
$$

where $m$ is the mass of electron, $\psi_{i}(\vec{r})$ is the natural orbitals, and $\nu_{i}$ is the occupation number of $\psi_{i}(\vec{r})$, respectively. The region with $n_{T}(\vec{r})>0$ can be regarded as an area where electrons can move freely in the meaning of classical mechanics, called the electronic drop region $R_{D}$, and the region with $n_{T}(\vec{r})<0$ means an area where electrons can move only with the quantum-mechanical tunneling effect, called the electronic atmosphere region $R_{A} . R_{D}$ and $R_{A}$ are divided by the electronic interface, the hypersurface of $n_{T}(\vec{r})=0 .{ }^{43}$ For the ${ }^{2} \mathrm{~S}$ ground state of $\mathrm{Li}$ atom, $R_{D}$ is distributed on the quiet neighborhood of nucleus with by far positive value and over a region where the distance from the nucleus is about 2.05.1 bohr. The practical electronic interface, $S$, can be regarded as the outer one at the distance of $5.1 \mathrm{bohr}$ from the 
nucleus. Electrons that occupy the core $1 s$ orbital are concentrated on the vicinity of the nucleus, so that the interface $S$ does not change so much by treating the valence electrons only. Therefore, the interface $S$ also means the hypersurface of the point where the spin kinetic-energy density is zero for the ground state of $\mathrm{Li}$ atom, the triplet states of $\mathrm{Li}_{2}$ molecule, and the quartet states of $\mathrm{Li}_{3}$ clusters, etc. The total electronic force density $\vec{F}^{S}(\vec{r})$ is represented as

$$
\vec{F}^{S}(\vec{r})=\vec{\tau}^{S}(\vec{r})+\vec{X}^{S}(\vec{r}),
$$

where $\vec{\tau}^{S}(\vec{r})$ and $\vec{X}^{S}(\vec{r})$ denote the electronic tension density and the electronic external force density, respectively. The superscript $S$ means that these densities originate in the velocity density operator $\hat{S}(\vec{r}) .{ }^{43}$ The $\vec{\tau}^{S}(\vec{r})$ has quantummechanical origin, and is defined as $\vec{\tau}^{S}(\vec{r})$ $={ }^{t}\left(\tau^{S 1}(\vec{r}), \tau^{S 2}(\vec{r}), \tau^{S 3}(\vec{r})\right)$ with

$$
\begin{aligned}
\tau^{S k}(\vec{r})= & \frac{\hbar^{2}}{4 m} \sum_{i} \nu_{i}\left(\psi_{i}^{*}(\vec{r}) \frac{\partial \Delta \psi_{i}(\vec{r})}{\partial x^{k}}-\frac{\partial \psi_{i}^{*}(\vec{r})}{\partial x^{k}} \Delta \psi_{i}(\vec{r})\right. \\
& \left.+\frac{\partial \Delta \psi_{i}^{*}(\vec{r})}{\partial x^{k}} \psi_{i}(\vec{r})-\Delta \psi_{i}^{*}(\vec{r}) \frac{\partial \psi_{i}(\vec{r})}{\partial x^{k}}\right)
\end{aligned}
$$

for $k=1,2,3$. The electric field due to interaction between electrons can be visualized properly by means of $\vec{\tau}^{S}(\vec{r})$. For stationary state, the $\vec{\tau}^{S}(\vec{r})$ balances with the electric field $\vec{E}(\vec{r})$ exerted on electron. ${ }^{43}$

For the ${ }^{3} \Sigma_{u}^{+}$state of $\mathrm{Li}_{2}$ molecule, it is observed that the MRCI potential-energy curve has a shallow minimum at $r$ $=7.452$ bohr, where $r$ denotes the distance between two $\mathrm{Li}$ atoms. The main configuration of the ${ }^{3} \Sigma_{u}^{+}$state is shown in Fig. 2. The dissociation limit of the ${ }^{3} \Sigma_{u}^{+}$state is ${ }^{2} S+{ }^{2} S$, where the electronic state can be represented by a single Slater determinant, but there is no minimum for the potential energy at the RHF level with the configuration of $\left(1 \sigma_{g}\right)^{2}\left(1 \sigma_{u}^{*}\right)^{2}\left(2 \sigma_{g}\right)^{1}\left(2 \sigma_{u}^{*}\right)^{1}$, as shown in Fig. 1(a). Accordingly, the consideration to excitations from the RHF configuration within the ${ }^{3} \Sigma_{u}^{+}$symmetry can stabilize the interaction between two Li atoms, even though a ratio of occupation on the excited configurations is small, as shown in Fig. 3. The kinetic-energy density and the tension density derived by Eqs. (1) and (3) for the ${ }^{3} \Sigma_{u}^{+}$state in the diatomic distance $r=11$ bohr at the MRCI level are shown in Fig. 4. For $r>11$ bohr, $R_{D}$ s around each atom are separated by $R_{A}$. Under this circumstance, the electronic state of system is almost equal to those of ${ }^{2} S \mathrm{Li}$ atoms at two positions, and each of valence electrons can make covalence only by the quantum-mechanical effects. For $r<$ about $11 \mathrm{bohr}, R_{D}$ has become one continuous region surrounding both two Li atoms. We have observed that the occupation on the excited configurations, which intensify the covalent interaction between Li atoms, starts to increase rapidly in the continuous $R_{D}$ as two Li atoms approach, as shown in Fig. 3. This result is considered as one of the evidences that valence electrons can move around both two Li atoms freely in the meaning of classical mechanics.

For the ${ }^{3} \Pi_{u}$ state, the MRCI potential-energy curve reaches a minimum at $r=4.887$ bohr. The dissociation limit of the ${ }^{3} \Pi_{u}$ state is ${ }^{2} S+{ }^{2} P$; valence $2 p$ electron in the ${ }^{2} P \mathrm{Li}$
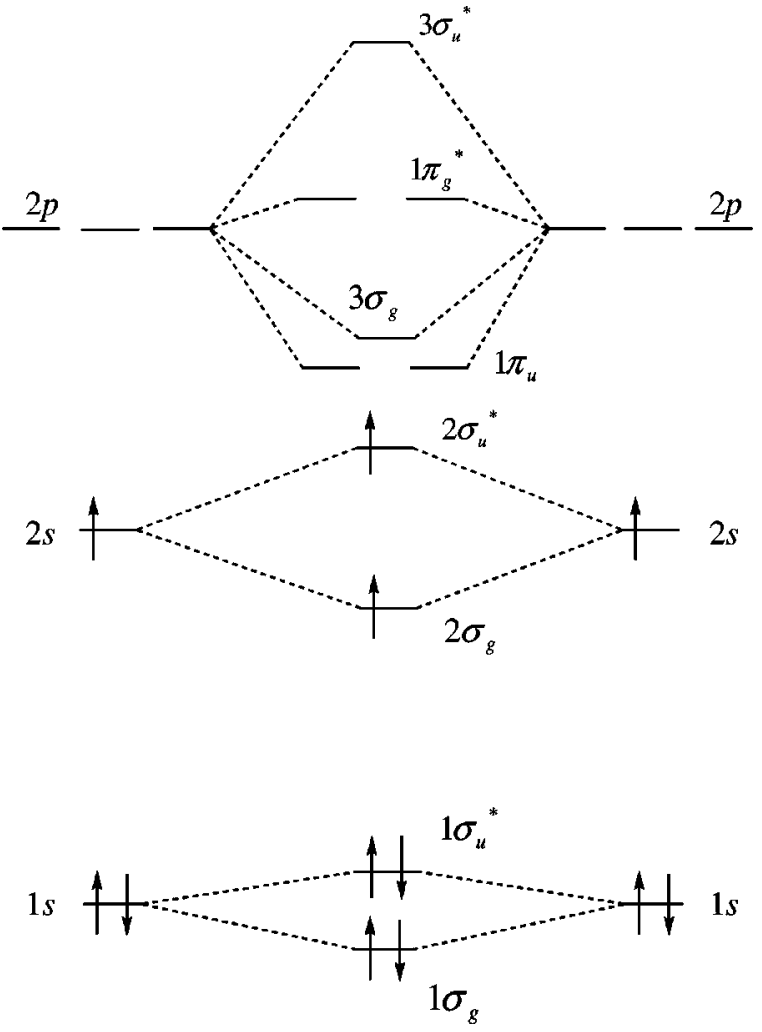

FIG. 2. Molecular orbital correlation diagram in the ${ }^{3} \Sigma_{u}^{+}$state of $\mathrm{Li}_{2}$ molecule.

atom stands vertical to the $\mathrm{Li}-\mathrm{Li}$ axis. The main configuration of the ${ }^{3} \Pi_{u}$ state at the vicinity of the minimum is $\left(1 \sigma_{g}\right)^{2}\left(1 \sigma_{u}^{*}\right)^{2}\left(2 \sigma_{g}\right)^{1}\left(1 \pi_{u}\right)^{1}$. The bond formation energy is very large because both of two valence electrons occupy bonding orbitals at the vicinity of the minimum for the ${ }^{3} \Pi_{u}$ state, contrary to the main configuration of the ${ }^{3} \Sigma_{u}^{+}$state.

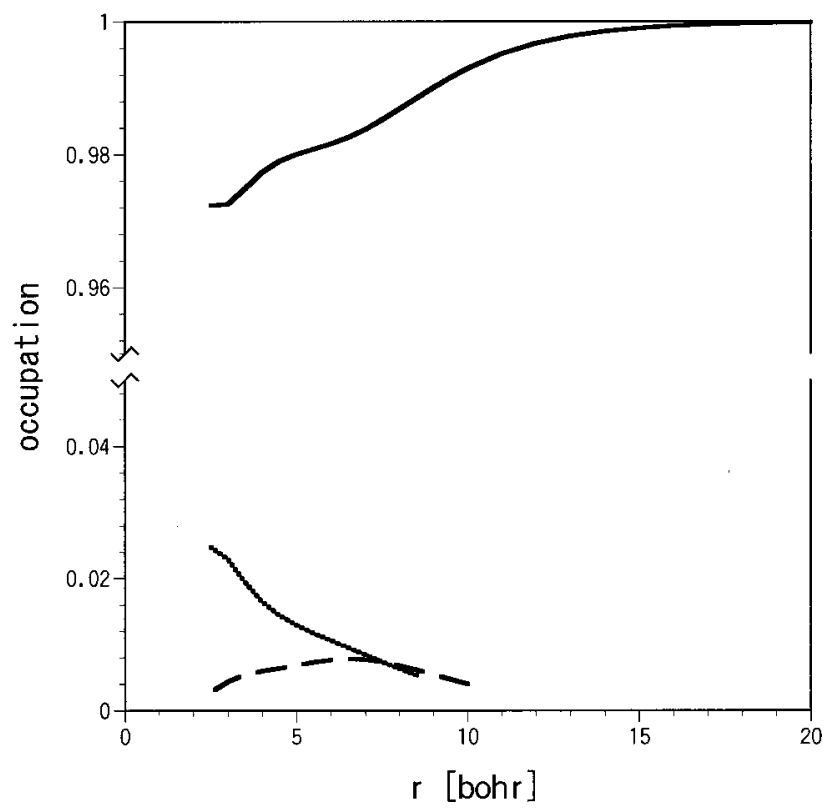

FIG. 3. Ratio of occupation on configurations for the ${ }^{3} \Sigma_{u}^{+}$state of $\mathrm{Li}_{2}$ molecule at the MRCI level. Solid line, dashed line, and dotted line denote the configurations of $\left(1 \sigma_{g}\right)^{2}\left(1 \sigma_{u}^{*}\right)^{2}\left(2 \sigma_{g}\right)^{1}\left(2 \sigma_{u}^{*}\right)^{1},\left(1 \sigma_{g}\right)^{2}\left(1 \sigma_{u}^{*}\right)^{2}$ $\left(3 \sigma_{g}\right)^{1}\left(3 \sigma_{u}^{*}\right)^{1}$, and $\left(1 \sigma_{g}\right)^{2}\left(1 \sigma_{u}^{*}\right)^{2}\left(1 \pi_{u}\right)^{1}\left(1 \pi_{g}^{*}\right)^{1}$, respectively. 

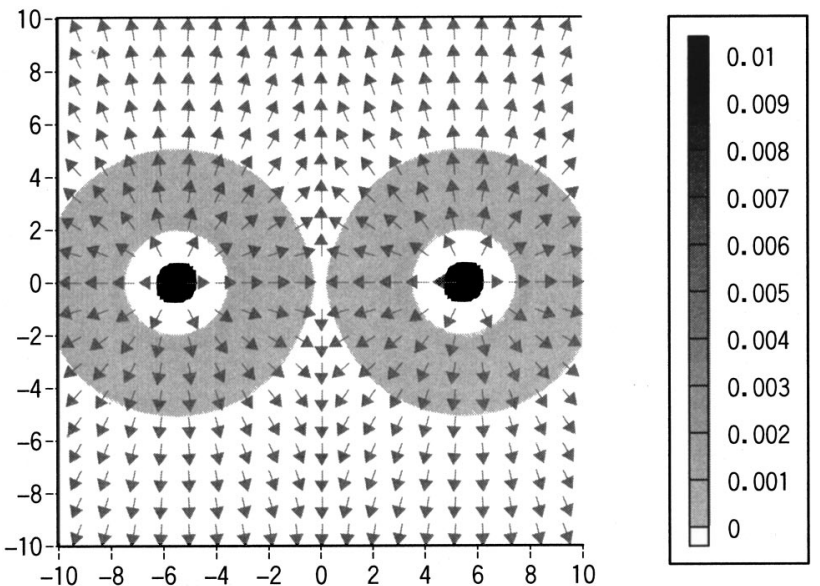

FIG. 4. Map of the kinetic-energy density $n_{T}(\vec{r})$ and the tension density $\vec{\tau}^{S}(\vec{r})$ (arrows) at the MRCI level for the ${ }^{3} \Sigma_{u}^{+}$state of $\mathrm{Li}_{2}$ molecule, where a diatomic distance is 11.0 bohr. Gray area is the electronic drop region.

Therefore, once a $\mathrm{Li}_{2}$ molecule becomes in the ${ }^{3} \Pi_{u}$ state, the strong $\mathrm{Li}-\mathrm{Li}$ bonding interaction is preserved, besides the fact that the transition between the ${ }^{3} \Sigma_{u}^{+}$state and the ${ }^{3} \Pi_{u}$ state is forbidden by symmetry. As shown in Fig. 5, the electronic state of the ${ }^{3} \Pi_{u}$ state cannot be represented by a single Slater determinant for large $r$, that is, $\left(1 \sigma_{g}\right)^{2}\left(1 \sigma_{u}^{*}\right)^{2}$ $\left(2 \sigma_{u}^{*}\right)^{1}\left(1 \pi_{g}^{*}\right)^{1}$ configuration makes a contribution of $50 \%$ occupation to the electronic state at the dissociation limit. Figure 6 shows the kinetic-energy density and the tension density for the ${ }^{3} \Pi_{u}$ state in $r=12$ bohr at the MRCI level. $R_{D}$ becomes one continuous region at $r \approx 12 \mathrm{bohr}$ by linking the electronic drop regions due to $2 p \pi$ electrons. Coulomb repulsion due to $1 \pi_{u}$ electron is relatively small, and therefore, the optimized length of $\mathrm{Li}-\mathrm{Li}$ bond is the shortest in Fig. 1.

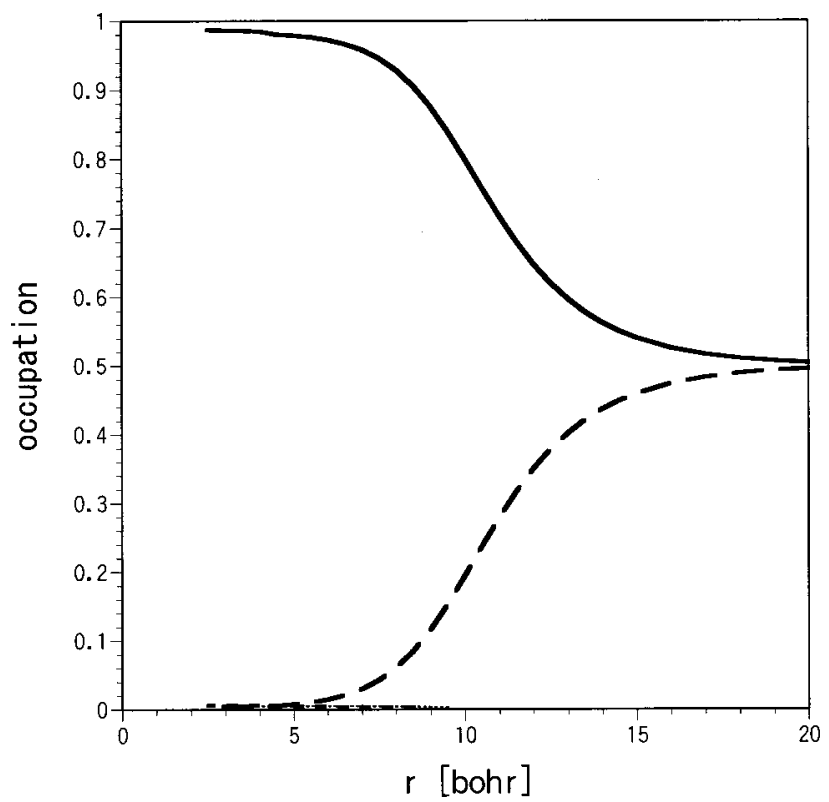

FIG. 5. Ratio of occupation on configurations for the ${ }^{3} \Pi_{u}$ state of $\mathrm{Li}_{2}$ molecule at the MRCI level. Solid line and dashed line denote the configurations of $\left(1 \sigma_{g}\right)^{2}\left(1 \sigma_{u}^{*}\right)^{2}\left(2 \sigma_{g}\right)^{1}\left(1 \pi_{u}\right)^{1}$ and $\left(1 \sigma_{g}\right)^{2}\left(1 \sigma_{u}^{*}\right)^{2}\left(2 \sigma_{u}^{*}\right)^{1}\left(1 \pi_{g}^{*}\right)^{1}$, respectively.
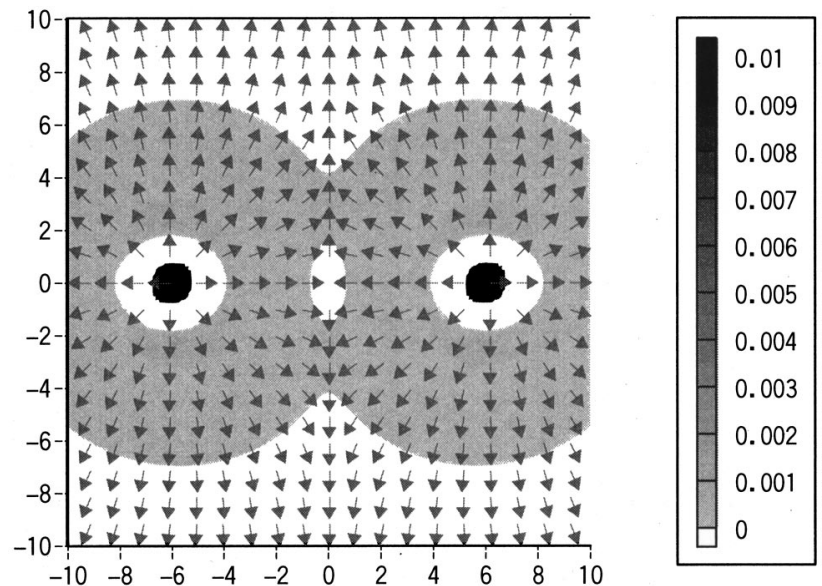

FIG. 6. Map of $n_{T}(\vec{r})$ and $\vec{\tau}^{S}(\vec{r})$ (arrows) at the MRCI level for the ${ }^{3} \Pi_{u}$ state of $\mathrm{Li}_{2}$ molecule, where a diatomic distance is 12.0 bohr. Map is set parallel to the $\pi$-plane.

For the ${ }^{3} \Sigma_{g}^{+}$state, the MRCI potential-energy curve reaches a minimum at $r=5.787 \mathrm{bohr}$. The dissociation limit of the ${ }^{3} \Sigma_{g}^{+}$state is ${ }^{2} S+{ }^{2} P$; valence $2 p$ electron in the ${ }^{2} P \mathrm{Li}$ atom stands parallel to the $\mathrm{Li}-\mathrm{Li}$ axis. The main configuration of the ${ }^{3} \Sigma_{g}^{+}$state at the vicinity of a minimum is $\left(1 \sigma_{g}\right)^{2}\left(1 \sigma_{u}^{*}\right)^{2}\left(2 \sigma_{g}\right)^{1}\left(3 \sigma_{g}\right)^{1}$. The bond formation energy of the ${ }^{3} \Sigma_{g}^{+}$state becomes large because of the same reason as that of the ${ }^{3} \Pi_{u}$ state. As shown in Fig. 7, the electronic state of the ${ }^{3} \Sigma_{g}^{+}$state cannot be also represented by a single Slater determinant for large $r$. As two $\mathrm{Li}$ atoms approach each other, a ratio of occupation on the minor $\left(1 \sigma_{g}\right)^{2}\left(1 \sigma_{u}^{*}\right)^{2}\left(2 \sigma_{u}^{*}\right)^{1}\left(3 \sigma_{u}^{*}\right)^{1}$ configuration, which makes contribution of $50 \%$ occupation at the dissociation limit, decreases more rapidly as compared with occupation on the minor configuration for the ${ }^{3} \Pi_{u}$ state. For very small $r$, a

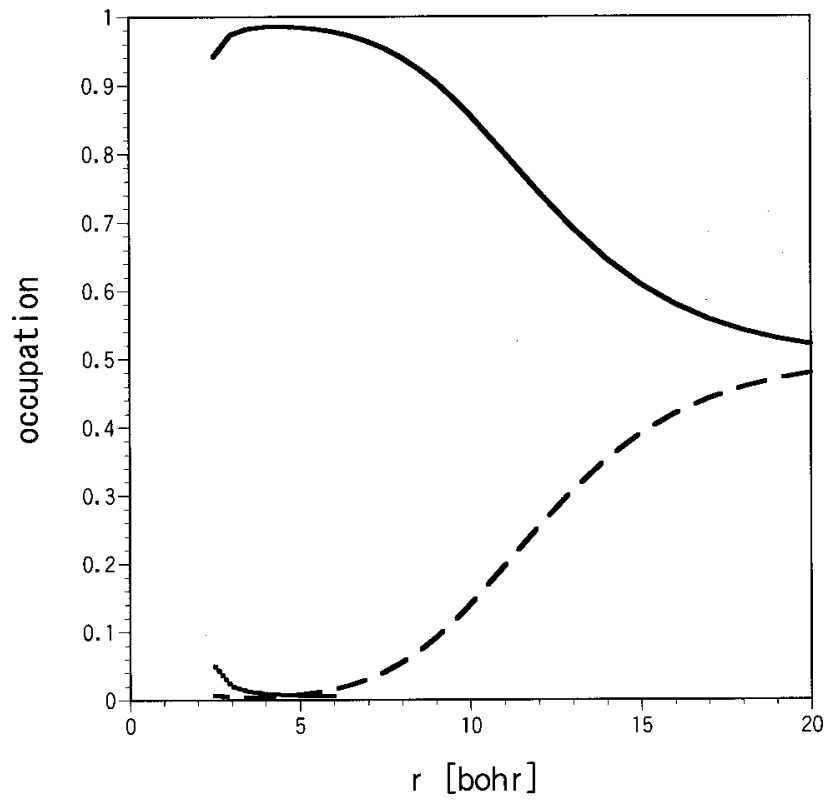

FIG. 7. Ratio of occupation on configurations for the ${ }^{3} \Sigma_{g}^{+}$state of $\mathrm{Li}_{2}$ molecule at the MRCI level. Solid line, dashed line, and dotted line denote the configurations of $\left(1 \sigma_{g}\right)^{2}\left(1 \sigma_{u}^{*}\right)^{2}\left(2 \sigma_{g}\right)^{1}\left(3 \sigma_{g}\right)^{1},\left(1 \sigma_{g}\right)^{2}\left(1 \sigma_{u}^{*}\right)^{2}\left(2 \sigma_{u}^{*}\right)^{1}$ $\left(3 \sigma_{u}^{*}\right)^{1}$, and $\left(1 \sigma_{g}\right)^{2}\left(1 \sigma_{u}^{*}\right)^{2}\left(1 \pi_{u}\right)^{1}\left(2 \pi_{u}\right)^{1}$, respectively. 

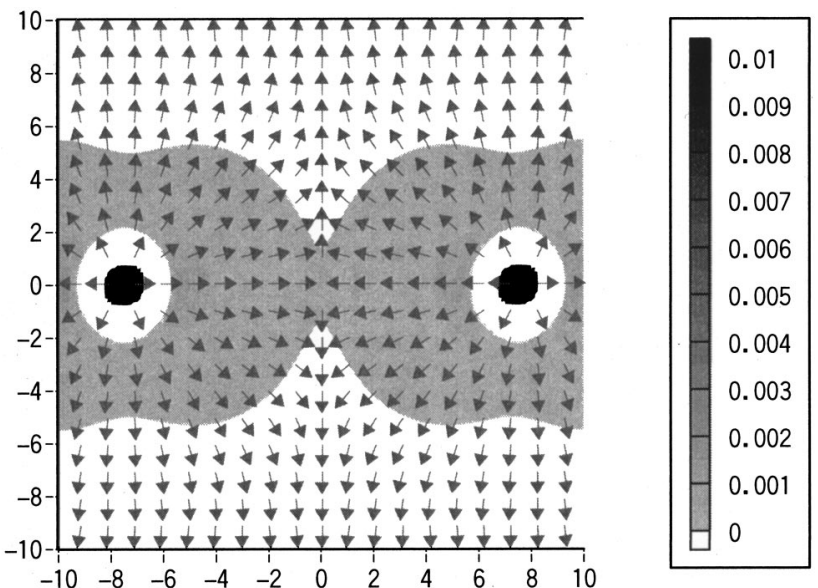

FIG. 8. Map of $n_{T}(\vec{r})$ and $\vec{\tau}^{S}(\vec{r})$ (arrows) at the MRCI level for the ${ }^{3} \Sigma_{g}^{+}$ state of $\mathrm{Li}_{2}$ molecule, where a diatomic distance is $15.0 \mathrm{bohr}$.

ratio of occupation on the main configuration is reduced to $80 \%$ because of the large repulsion due to $2 \sigma_{g}$ and $3 \sigma_{g}$ electrons, and the occupation of $\left(1 \sigma_{g}\right)^{2}\left(1 \sigma_{u}^{*}\right)^{2}\left(1 \pi_{u}\right)^{1}$ $\left(2 \pi_{u}\right)^{1}$ configuration can be observed. Figure 8 shows the kinetic-energy density and the tension density for the ${ }^{3} \Sigma_{g}^{+}$ state in $r=15$ bohr at the MRCI level. $R_{D}$ becomes one continuous region at $r \approx 15 \mathrm{bohr}$, and the increase of interaction by the linkage of $R_{D}$ at the earlier stage of bond formation can explain the result that a ratio of occupation on the minor configuration decreases more rapidly.

For the ${ }^{3} \Pi_{g}$ state, it is noteworthy that the MRCI potential-energy curve has no minimum. We have treated each of two electronic configurations, $\left(1 \sigma_{g}\right)^{2}\left(1 \sigma_{u}^{*}\right)^{2}$ $\left(2 \sigma_{g}\right)^{1}\left(1 \pi_{g}^{*}\right)^{1}$ and $\left(1 \sigma_{g}\right)^{2}\left(1 \sigma_{u}^{*}\right)^{2}\left(2 \sigma_{u}^{*}\right)^{1}\left(1 \pi_{u}\right)^{1}$, for the RHF configuration. The SCF energy of the former configuration is lower than that of the latter for $r<13 \mathrm{bohr}$, but the difference in energy is not so large. Both of the RHF potential-energy curves have a minimum because the electronic state of the ${ }^{3} \Pi_{g}$ state for large $r$ cannot be represented by a single Slater determinant, and as a result, the RHF energies are raised extremely for large $r$. As shown in Fig. 9, the main configuration of the ${ }^{3} \Pi_{g}$ state changes place abruptly in the range from $r \approx 3 \mathrm{bohr}$ to $r \approx 4 \mathrm{bohr}$. For $r$ $>4 \mathrm{bohr}$, the former configuration is main, though a ratio of the occupation is reduced to $50 \%$ at large $r$. On the other hand, for $r<3 \mathrm{bohr}$, the former configuration is destabilized by the large repulsion due to $2 \sigma_{g}$ electron, just as a ratio of occupation on the main configuration is reduced for small $r$ in the ${ }^{3} \Sigma_{g}^{+}$state, and then the latter configuration becomes main. Figure 10 shows the kinetic-energy density and the tension density for the ${ }^{3} \Pi_{g}$ state in $r=4.5$ bohr at the MRCI level. $R_{D}$ becomes one continuous region at $r \approx 11 \mathrm{bohr}$, and when the Li-Li distance is relatively small, $R_{D}$ spreads out widely for $r<7$ bohr. However, some sharp cracks of $R_{A}$ are observed in $R_{D}$, and therefore, it is expected easily that covalent interactions over the crack of $R_{A}$ are very hard to achieve. This forecast for weak covalent interactions leads to the fact that the potential-energy curve is repulsive.

Thus, it is found that the shape of $R_{D}$ depends strongly on the electronic state and represents the characteristics of

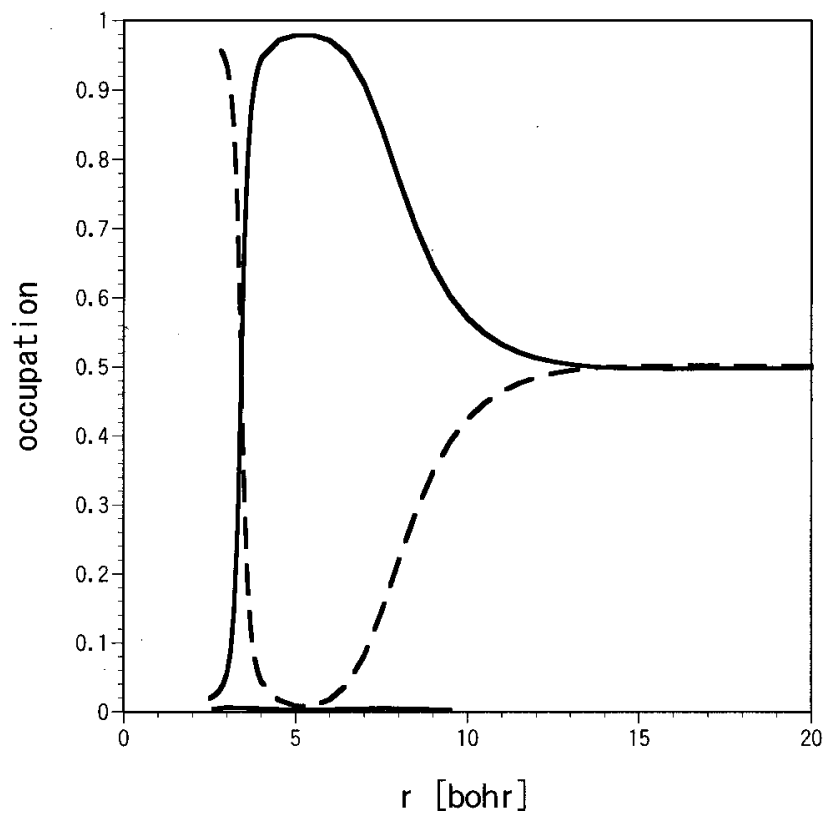

FIG. 9. Ratio of occupation on configurations for the ${ }^{3} \Pi_{g}$ state of $\mathrm{Li}_{2}$ molecule at the MRCI level. Solid line and dashed line denote the configurations of $\left(1 \sigma_{g}\right)^{2}\left(1 \sigma_{u}^{*}\right)^{2}\left(2 \sigma_{g}\right)^{1}\left(1 \pi_{g}^{*}\right)^{1}$ and $\left(1 \sigma_{g}\right)^{2}\left(1 \sigma_{u}^{*}\right)^{2}\left(2 \sigma_{u}^{*}\right)^{1}\left(1 \pi_{u}\right)^{1}$, respectively.

interaction clearly. When the separated two $R_{D} \mathrm{~S}$ connect to each other for $\mathrm{Li}_{2}$ molecule, ratios of occupation on configurations change rapidly not only in Fig. 3 but also in Figs. 5, 7, and 9. The $\vec{\tau}^{S}(\vec{r})$ gives new images of microscopic electronic stresses.

\section{B. Quartet states of $\mathrm{Li}_{3}$ clusters and propagation of cluster}

In this section, the electronic states of the quartet $\mathrm{Li}_{3}$ clusters shall be discussed. We have investigated the potential-energy curves for the colinear structure and the triangular structure. Figure 11 shows potential-energy curves with respect to the $\mathrm{Li}-\mathrm{Li}$ distance $r$ for the ${ }^{3} \Sigma_{u}^{+} \mathrm{Li}_{2}$ part in
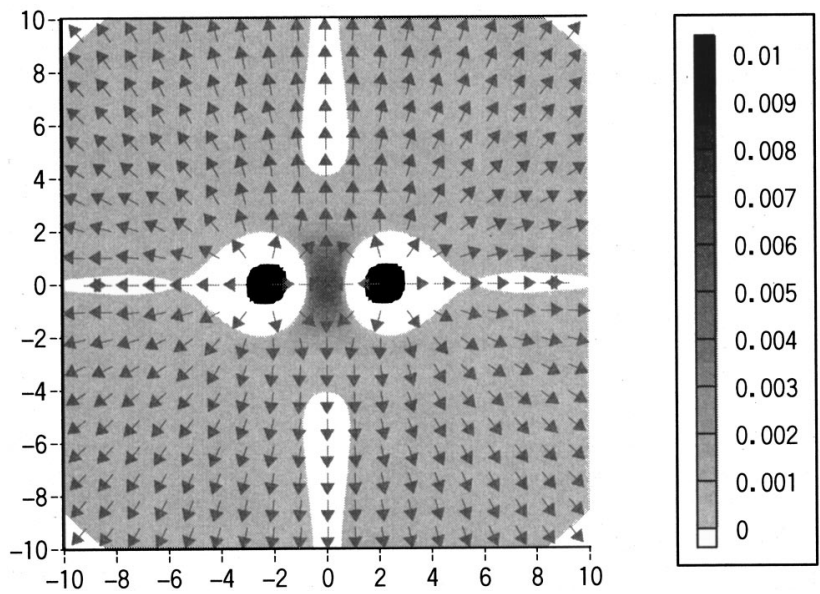

FIG. 10. Map of $n_{T}(\vec{r})$ and $\vec{\tau}^{S}(\vec{r})$ (arrows) at the MRCI level for the ${ }^{3} \Pi_{g}$ state of $\mathrm{Li}_{2}$ molecule, where a diatomic distance is 4.5 bohr. Map is set parallel to the $\pi$-plane. 


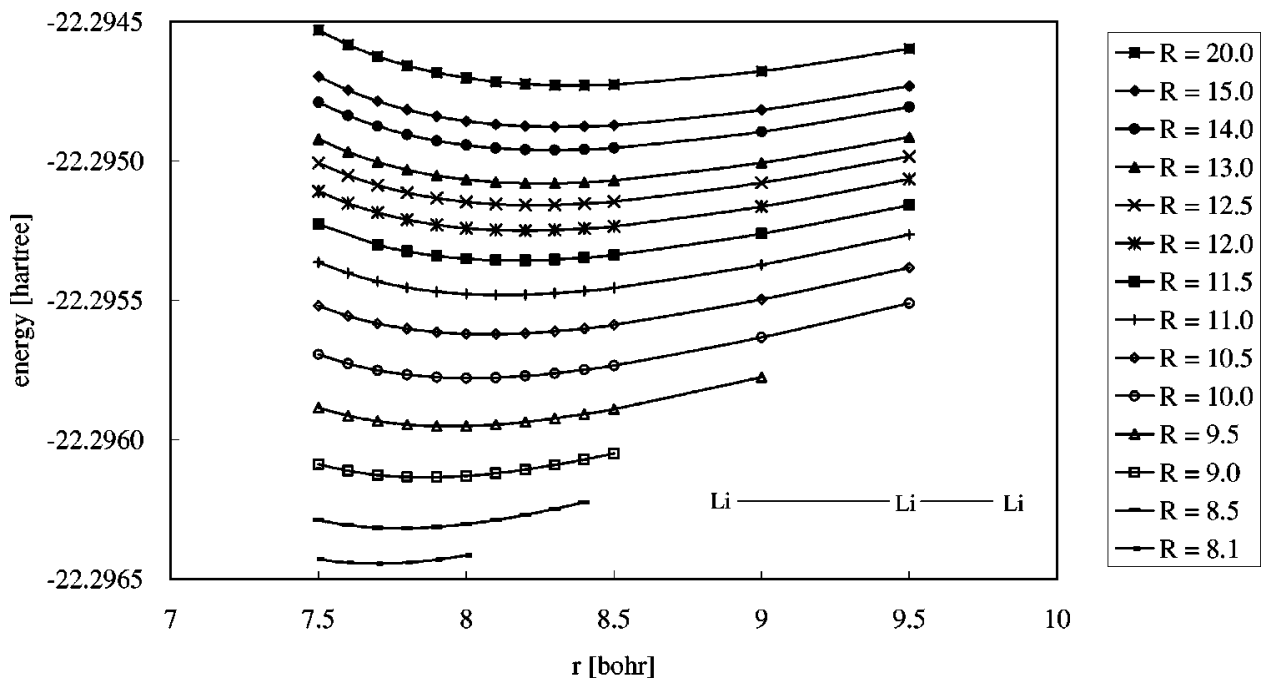

FIG. 11. Potential-energy curves for the ${ }^{4} \Sigma^{+}$colinear $\mathrm{Li}_{3}$ cluster system with respect to the $\mathrm{Li}-\mathrm{Li}$ distance $r$ for the ${ }^{3} \Sigma_{u}^{+} \mathrm{Li}_{2}$ part at the MRCI level. the ${ }^{4} \Sigma^{+}$colinear $\mathrm{Li}_{3}$ cluster. It is found that the ${ }^{4} \Sigma^{+} \mathrm{Li}_{3}$ cluster becomes more stable as the distance $R$ between the ${ }^{2} S \mathrm{Li}$ atom and the nearer $\mathrm{Li}$ atom of the ${ }^{3} \Sigma_{u}^{+} \mathrm{Li}_{2}$ molecule shortens. Simultaneously, it is observed that the optimized Li-Li distance for the ${ }^{3} \Sigma_{u}^{+} \mathrm{Li}_{2}$ part with fixed $R$ becomes shorter as the ${ }^{2} S \mathrm{Li}$ atom approaches. In the computational level in this section, the optimized Li-Li length and the bond formation energy of the ${ }^{3} \Sigma_{u}^{+} \mathrm{Li}_{2}$ molecule are respectively 8.372 bohr and $0.027 \mathrm{eV}$. These results are different from those of the computational level in the previous section to some extent, but there is no problem discussing the qualitative character of interactions among $\mathrm{Li}_{3}$ system. The most stable geometry of the ${ }^{4} \Sigma^{+}$colinear $\mathrm{Li}_{3}$ cluster becomes the $D_{\infty h}$ structure where $r=R=7.589$ bohr. Stabilization energy from the ${ }^{2} \mathrm{~S} \mathrm{Li}+{ }^{3} \Sigma_{u}^{+} \mathrm{Li}_{2}$ system is $0.050 \mathrm{eV}$. That is, the decrease in energy due to the linear propagation from $\mathrm{Li}_{2}$ to $\mathrm{Li}_{3}$ by the ground state of $\mathrm{Li}$ atom in the high-spin electronic state is as large as the bond formation energy of the ${ }^{3} \Sigma_{u}^{+} \mathrm{Li}_{2}$ molecule.

Figure 12 shows potential-energy curves with respect to $r$ for the ${ }^{3} \Sigma_{u}^{+} \mathrm{Li}_{2}$ part in the triangular $\mathrm{Li}_{3}$ cluster of the ${ }^{4} B_{2}$ state. In the triangular structures, potential energy is reduced more extremely as the distance $R$ between the ${ }^{2} S \mathrm{Li}$ atom and the midpoints of $\mathrm{Li}$ atoms of the ${ }^{3} \Sigma_{u}^{+} \mathrm{Li}_{2}$ molecule shortens, because the single occupied MO (SOMO) of the ${ }^{3} \Sigma_{u}^{+} \mathrm{Li}_{2}$ part widely overlaps the $2 p$ unoccupied atomic orbital at both ends of the $p$-lobe. The most stable geometry of the ${ }^{4} B_{2}$ triangular $\mathrm{Li}_{3}$ cluster becomes the $D_{3 h}$ regular triangular structure where $r=6.090$ bohr and $R=5.274$ bohr. Stabilization energy from the ${ }^{2} S \mathrm{Li}+{ }^{3} \Sigma_{u}^{+} \mathrm{Li}_{2}$ system is 0.327 $\mathrm{eV}$, which is quite larger than that of the colinear cluster. For the triangular structure, the first excited state is the ${ }^{4} A_{2}$ state, where dissociation limit between $\mathrm{Li}$ and $\mathrm{Li}_{2}$ is ${ }^{2} P+{ }^{3} \Sigma_{u}^{+}$. Potential energy of the most stable structure of the ${ }^{4} A_{2}$ state ( $r=7.446 \mathrm{bohr}$ and $R=3.744 \mathrm{bohr}$ ) is higher by $0.450 \mathrm{eV}$ than that of the most stable ${ }^{4} B_{2} \mathrm{Li}_{3}$ cluster, that is, the ${ }^{4} A_{2}$ triangular $\mathrm{Li}_{3}$ cluster is more unstable than the ${ }^{2} \mathrm{~S} \mathrm{Li}+{ }^{3} \Sigma_{u}^{+}$ $\mathrm{Li}_{2}$ system.

Here, we shall prove that the triangular $\mathrm{Li}_{3}$ cluster in the lowest quartet state has the $D_{3 h}$ symmetry. Figure 13 displays the global lowest ${ }^{4} A^{\prime}$ potential energy surface for $\mathrm{Li}_{3}$ cluster with the $C_{s}$ symmetry in the vicinity of the $D_{3 h}$ regu-

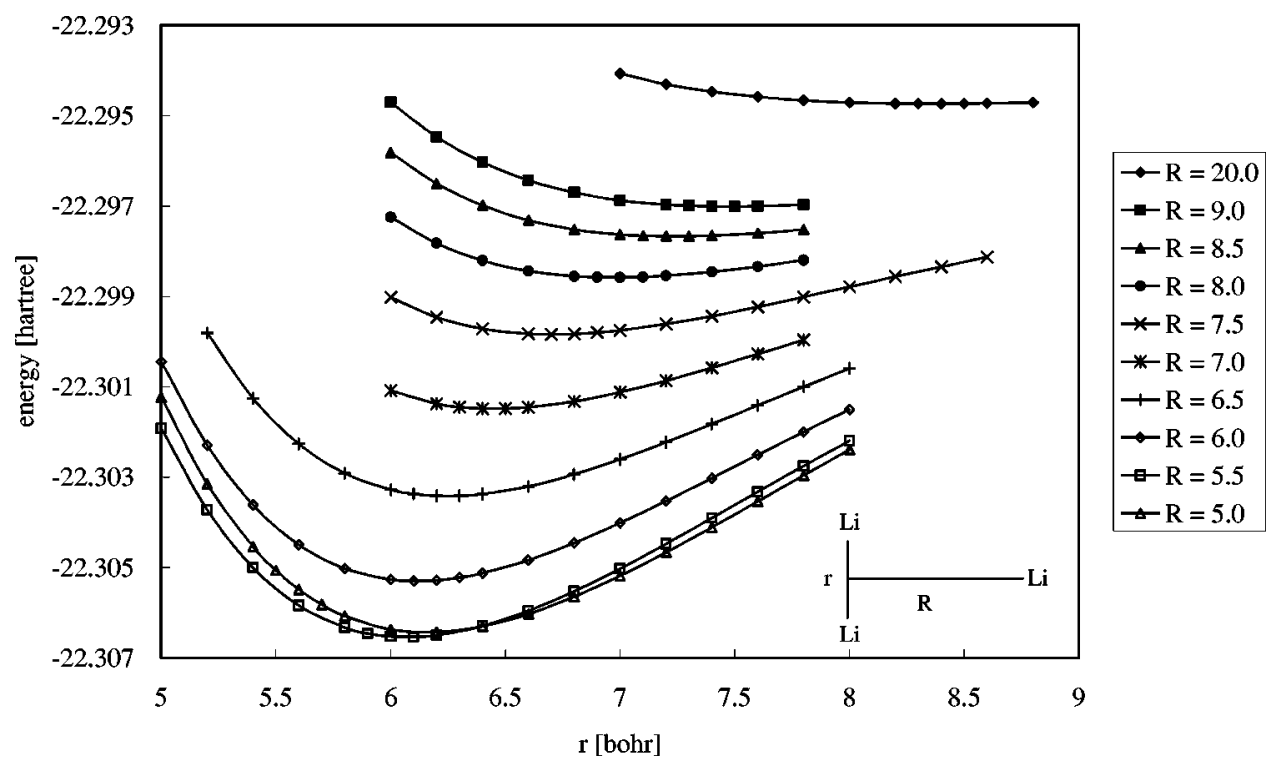

FIG. 12. Potential-energy curves for the ${ }^{4} B_{2}$ triangular $\mathrm{Li}_{3}$ cluster system with respect to the $\mathrm{Li}-\mathrm{Li}$ distance $\mathrm{r}$ for the ${ }^{3} \Sigma_{u}^{+} \mathrm{Li}_{2}$ part at the MRCI level. 


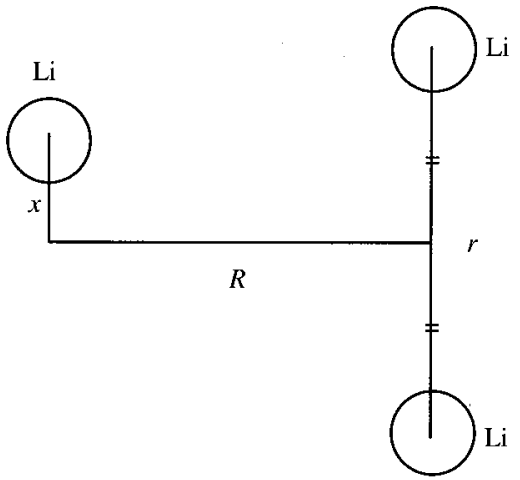

(a)

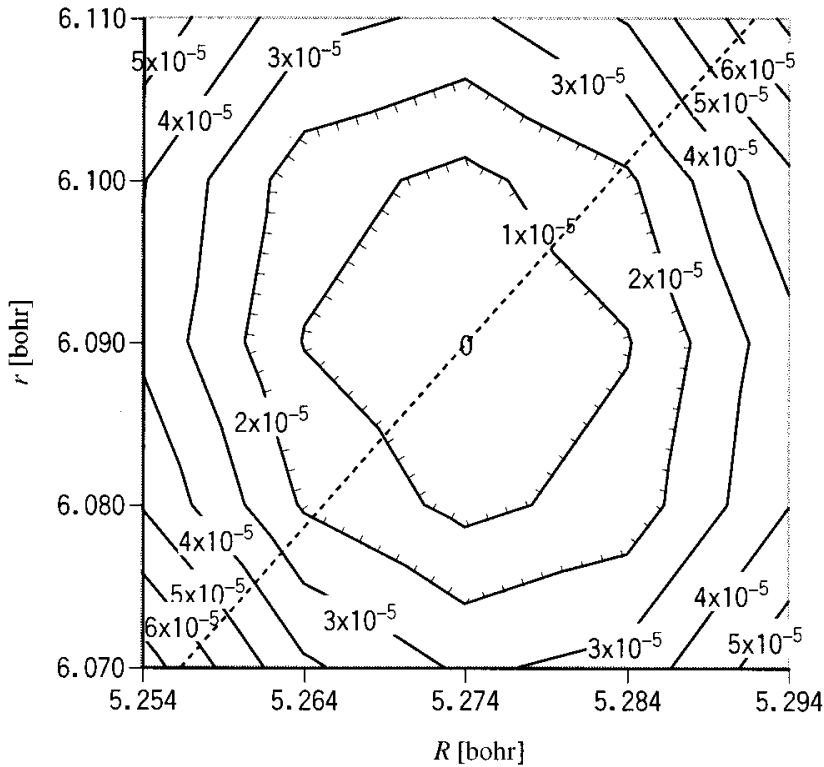

(b)

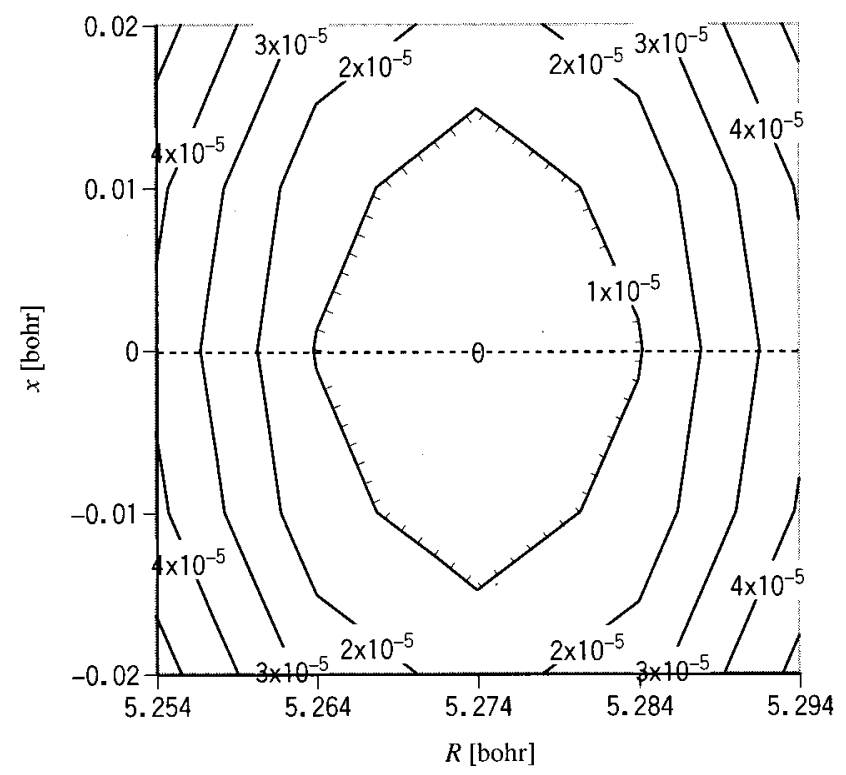

(c)

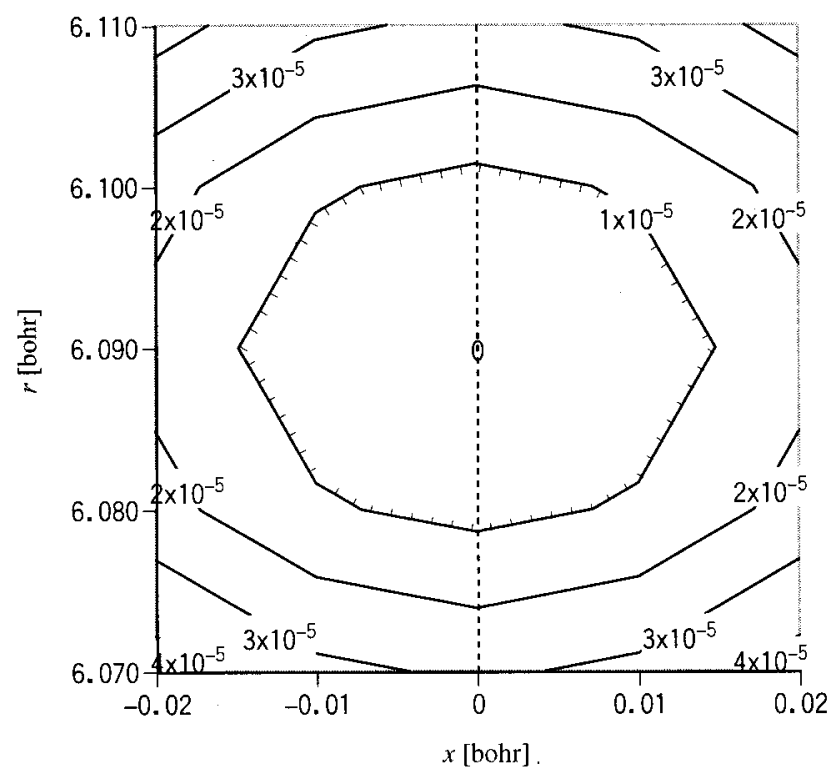

(d)

FIG. 13. Global lowest ${ }^{4} A^{\prime}$ potential-energy surface for $\mathrm{Li}_{3}$ cluster with the $C_{s}$ symmetry: (a) displacements of the $C_{s}$ structure, and potential-energy surface maps with keeping (b) $x=0$, (c) $r=6.090 \mathrm{bohr}$, and (d) $R=5.274 \mathrm{bohr}$, respectively. Contours are drawn every $1 \times 10^{-5} \mathrm{eV}$. Map (b) denotes the ${ }^{4} B_{2}$ potential-energy surface with keeping the $C_{2 v}$ symmetry, and the electronic state is represented as ${ }^{4} A_{2}^{\prime}$ with the $D_{3 h}$ symmetry on the dashed line. On the dashed line in maps (c) and (d), the electronic state is represented as ${ }^{4} B_{2}$ with the $C_{2 v}$ symmetry.

lar triangular structure where $r=6.090 \mathrm{bohr}$ and $R$ $=5.274$ bohr. At $x=0$, the cluster has the $C_{2 v}$ symmetry, and then, the electronic state is represented as ${ }^{4} B_{2}$. It is clearly found that the $D_{3 h}$ structure without any structural distortion is located at the minimum of the global lowest ${ }^{4} A^{\prime}$ potentialenergy surface. In the $D_{3 h}$ structure, the degenerate MOs can be observed, and the occupation on each one of the degenerate MOs is identical with that on the other for the ${ }^{4} A_{2}^{\prime}$ state $\left({ }^{4} B_{2}\right.$ state in the $C_{2 v}$ symmetry), as shown in Fig. 14(a). Therefore, the Jahn-Teller distortion, which is associated with removal of the MO degeneracy, cannot occur in the ${ }^{4} A_{2}^{\prime}$ state with the $D_{3 h}$ symmetry. On the other hand, it has been reported that the symmetry of the most stable structure of $\mathrm{Li}_{3}$

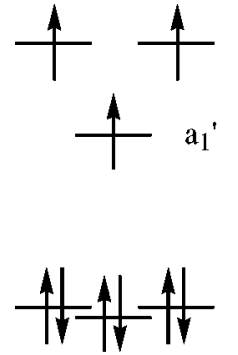

(a)
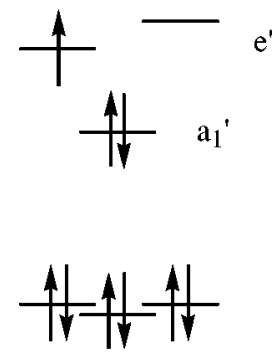

(b)
FIG. 14. Electronic configurations for the $D_{3 h} \mathrm{Li}_{3}$ cluster in (a) the highspin and (b) the low-spin states. 


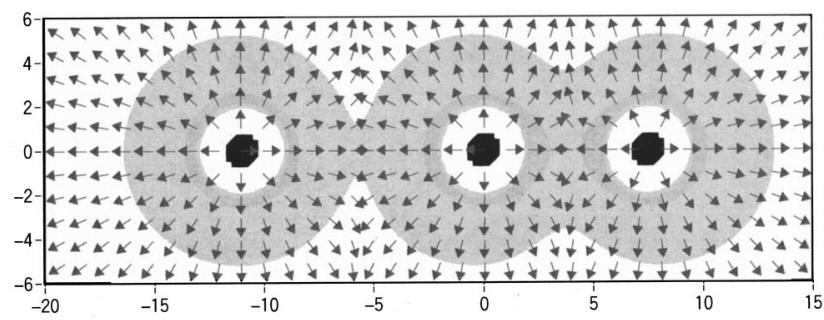

(a)

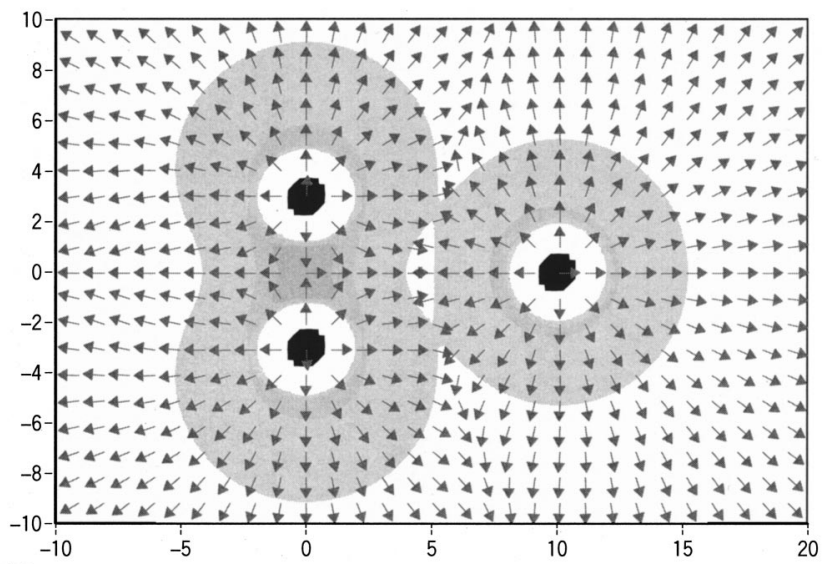

(b)

FIG. 15. Maps of $n_{T}(\vec{r})$ and $\vec{\tau}^{S}(\vec{r})$ (arrows) at the MRCI level for (a) the ${ }^{4} \Sigma^{+}$colinear $\mathrm{Li}_{3}$ cluster with $R=11.0 \mathrm{bohr}, r=7.5 \mathrm{bohr}$ and (b) the ${ }^{4} B_{2}$ triangular $\mathrm{Li}_{3}$ cluster with $R=10.0 \mathrm{bohr}, r=6.0 \mathrm{bohr}$.

cluster in the low-spin electronic state is not $D_{3 h}$ but $C_{2 v}$ because of the Jahn-Teller effect: the largest bond angle is about $73^{\circ} .{ }^{31-38}$ The MO degeneracy has been removed by occupation of the doublet state as shown in Fig. 14(b), and therefore, the Jahn-Teller distortion is effective for the triangular structure in the low-spin electronic state.

Examples of $n_{T}(\vec{r})$ and $\vec{\tau}^{S}(\vec{r})$ for the ${ }^{4} \Sigma^{+}$colinear and the ${ }^{4} B_{2}$ triangular structures are shown in Fig. 15. The shape of $R_{D}$ indicates clearly that these electronic states are established by joining the ${ }^{2} S$ state of $\mathrm{Li}$ atom to the ${ }^{3} \Sigma_{u}^{+}$state of $\mathrm{Li}_{2}$ molecule. For the ${ }^{4} \Sigma^{+}$state, it is observed that ${ }^{2} S \mathrm{Li}$ atom interacts with $\mathrm{Li}_{2}$ molecule just as two $\mathrm{Li}$ atoms do with each other in the ${ }^{3} \Sigma_{u}^{+} \mathrm{Li}_{2}$ molecule. Thus, the stabilization energy due to formation of a ${ }^{4} \Sigma^{+}$colinear $\mathrm{Li}_{3}$ cluster from a $\mathrm{Li}$ atom and a ${ }^{3} \Sigma_{u}^{+} \mathrm{Li}_{2}$ molecule results in as large as the formation energy of the ${ }^{3} \Sigma_{u}^{+} \mathrm{Li}_{2}$ molecule. For the ${ }^{4} B_{2}$ state, $R_{D}$ around the ${ }^{2} S \mathrm{Li}$ atom and $R_{D}$ around the ${ }^{3} \Sigma_{u}^{+} \mathrm{Li}_{2}$ molecule touch and form one continuous region at $R$ $\approx 10$ bohr. The connection occurs not on the perpendicular to the $\mathrm{Li}_{2}$ axis but at two points on equilateral lines of the triangle. When the Li atom draws near to the $\mathrm{Li}_{2}$ molecule, $R_{D}$ has unified completely as it surrounds the frame of triangle. In this $R_{D}$, three valence electrons can move around the triangle freely, and remarkable stabilization of the system can be estimated due to delocalization of valence electrons.

As a result, $\mathrm{a} \mathrm{Li}_{3}$ cluster has a triangular structure rather than a colinear one in high-spin electronic state. Next, we shall discuss formation of quintet $\mathrm{Li}_{4}$ clusters from the ${ }^{4} A_{2}^{\prime}$ triangular $\mathrm{Li}_{3}$ cluster. It has been reported that the $D_{2 h}$ rhombic planar structure is more stable than the $C_{3 v}$ regular tri-

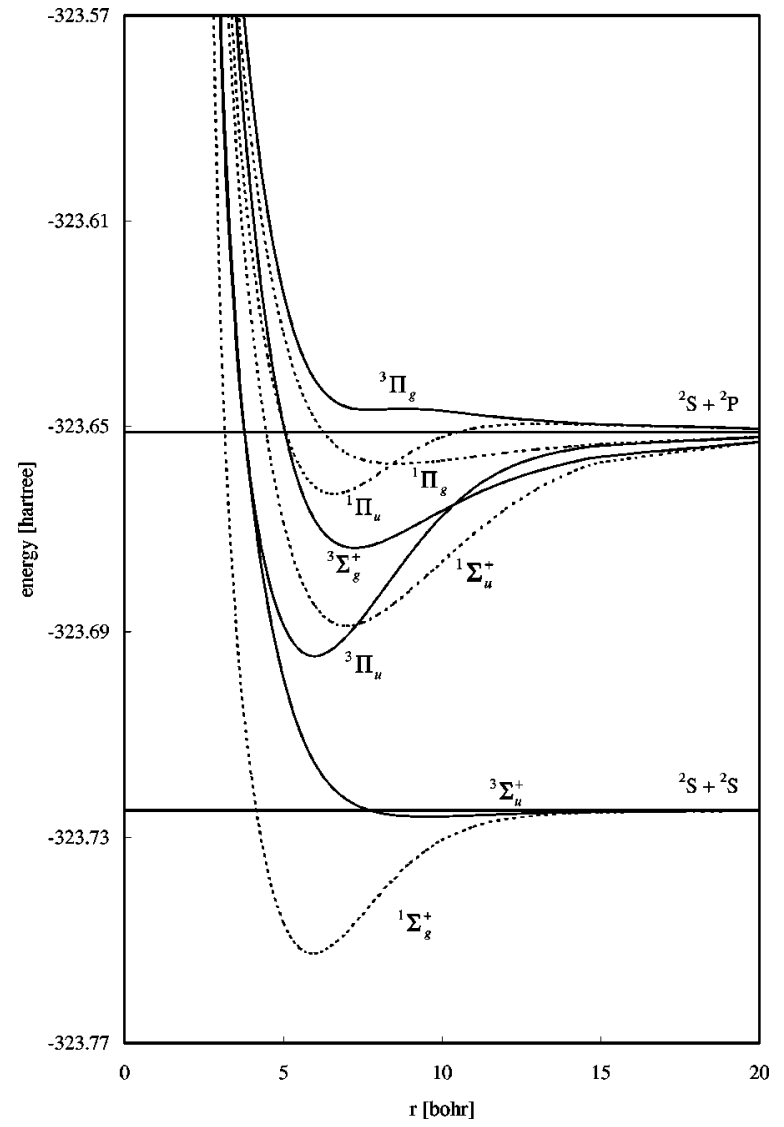

FIG. 16. Potential-energy curves for each electronic state of $\mathrm{Na}_{2}$ molecule at the MRCI levels. Curves by solid line and dashed line, respectively, denote the triplet states and the singlet states.

angular pyramidal structure for the low-spin electronic state of $\mathrm{Li}_{4}$ clusters. ${ }^{31-38}$ However, for the quintet electronic state, the most stable structure of $\mathrm{Li}_{4}$ cluster is the $T_{d}$ regular tetrahedral structure, where the electronic state is ${ }^{5} A_{1}$ and the $\mathrm{Li}-\mathrm{Li}$ distance is $5.822 \mathrm{bohr}$. The $T_{d}$ structure has the triple degenerate MOs, and the occupation on each one of the degenerate MOs is identical with that on the others for the quintet ${ }^{5} A_{1}$ state. Therefore, the Jahn-Teller distortion is ineffective, similarly as in the quartet $D_{3 h}$ structure. Stabilization energy from the ${ }^{2} S \mathrm{Li}+{ }^{4} B_{2} \mathrm{Li}_{3}$ system is $0.773 \mathrm{eV}$, which is bigger than twice of that of the most stable $\mathrm{Li}_{3}$ cluster. Potential energies of stable $D_{2 h}$ rhombic structures, ${ }^{5} A_{u}$ and ${ }^{5} B_{3 g}$, are higher by 0.453 and $0.712 \mathrm{eV}$ than that of the $T_{d}$ structure, respectively. Accordingly, it is expected that even the most initial stage of cluster propagation would proceed not to a board but to a sphere, and there, stabilization energy due to taking in a $\mathrm{Li}$ atom is raised as the number of atoms in $\mathrm{Li}_{n}$ cluster increases for $n=1,2$, and 3.

\section{High-spin states of sodium clusters}

Figure 16 shows the potential-energy curves for each electronic state of $\mathrm{Na}_{2}$ molecule at the MRCI level. Similarly as $\mathrm{Li}_{2}$ molecule, minimum on the potential-energy curve of ${ }^{3} \Sigma_{u}^{+}$state appears at the MCSCF and the MRCI levels. The optimized $\mathrm{Na}-\mathrm{Na}$ length and the bond formation-energy are listed in Table II. Compared with the potential-energy curves of $\mathrm{Li}_{2}$ molecule, it is clearly found that (1) the bond forma- 
TABLE II. Optimized length and bond formation energy of $\mathrm{Na}_{2}$ at the MRCI level with Roos' extended Gaussian basis set.

\begin{tabular}{lcccc}
\hline \hline & & Bond & \multicolumn{2}{c}{ Absolute energy (Hartree) } \\
\cline { 4 - 5 } & $\begin{array}{c}\text { Optimized } \\
\text { State }\end{array}$ & $\begin{array}{c}\text { cormation } \\
\text { length (bohr) }\end{array}$ & $\begin{array}{c}\text { in optimized } \\
\text { energy (eV) }\end{array}$ & $\begin{array}{c}\text { length } \\
\text { in dissociation } \\
\text { limit }\end{array}$ \\
\hline${ }^{1} \Sigma_{g}^{+}$ & 5.922 & 0.758 & -323.75257 & $-323.72471\left({ }^{2} S+{ }^{2} S\right)$ \\
${ }^{3} \Sigma_{u}^{+}$ & 9.512 & 0.034 & -323.72596 & $-323.72471\left({ }^{2} S+{ }^{2} S\right)$ \\
${ }^{3} \Pi_{u}$ & 5.968 & 1.185 & -323.69471 & $-323.65115\left({ }^{2} S+{ }^{2} P\right)$ \\
${ }^{1} \Sigma_{u}^{+}$ & 6.999 & 1.025 & -323.68883 & $-323.65115\left({ }^{2} S+{ }^{2} P\right)$ \\
${ }^{3} \Sigma_{g}^{+}$ & 7.258 & 0.613 & -323.67366 & $-323.65115\left({ }^{2} S+{ }^{2} P\right)$ \\
${ }^{1} \Pi_{u}$ & 6.591 & 0.327 & -323.66315 & $-323.65115\left({ }^{2} S+{ }^{2} P\right)$ \\
${ }^{1} \Pi_{g}$ & 8.543 & 0.166 & -323.65726 & $-323.65115\left({ }^{2} S+{ }^{2} P\right)$ \\
${ }^{3} \Pi_{g}$ & $\cdots$ & $\cdots$ & $\cdots$ & $-323.65115\left({ }^{2} S+{ }^{2} P\right)$ \\
\hline \hline
\end{tabular}

tion energies for each electronic state of $\mathrm{Na}_{2}$ molecule are less than the corresponding electronic state of $\mathrm{Li}_{2}$; (2) the potential-energy curve of ${ }^{3} \Pi_{u}$ state does not cross that of ${ }^{3} \Sigma_{u}^{+}$state in $\mathrm{Na}_{2}$. The former means that the interaction between $\mathrm{Na}$ atoms is smaller than the interaction between $\mathrm{Li}$ atoms, and the latter is caused by the high excitation energy of $\mathrm{Na}$ atom, from ${ }^{2} S$ state to ${ }^{2} P$ state. As a result, a quite large absolute energy difference between the ${ }^{3} \Sigma_{u}^{+}$state and the ${ }^{3} \Pi_{u}$ state appears in the $\mathrm{Na}_{2}$ molecule. Under the strong magnetic field, the ${ }^{2} P$ state of atom and the ${ }^{3} \Pi_{u}$ state of diatomic molecule suffer from the Zeeman effect, and there, it is expected that the ${ }^{3} \Pi_{u}$ state can be selected more easily for $\mathrm{Li}_{2}$ molecule as a deep potential well, because the absolute ${ }^{3} \Sigma_{u}^{+}-{ }^{3} \Pi_{u}$ energy difference is small. However, it would be hard to take the $\mathrm{Na}_{2}$ molecule in the ${ }^{3} \Pi_{u}$ state because the ${ }^{3} \Sigma_{u}^{+}-{ }^{3} \Pi_{u}$ energy difference is large. For the detailed discussion, the electronic-state calculations of species in strong magnetic field are required. ${ }^{57-60}$

Moreover, we have investigated the optimized structures of colinear and triangular $\mathrm{Na}_{3}$ clusters in the quartet state. Similarly as the $\mathrm{Li}_{3}$ clusters, the most stable state of the colinear $\mathrm{Na}_{3}$ and the triangular $\mathrm{Na}_{3}$ is ${ }^{4} \Sigma^{+}$and ${ }^{4} B_{2}$, respectively. The colinear $\mathrm{Na}_{3}$ cluster becomes the $D_{\infty h}$ structure by geometry optimization where $r=R=10.192 \mathrm{bohr}$, and the stabilization energy from the ${ }^{2} S \mathrm{Na}+{ }^{3} \Sigma_{u}^{+} \mathrm{Na}_{2}$ system is $0.016 \mathrm{eV}$. It is found that to the linear propagation from $\mathrm{Na}_{2}$ to $\mathrm{Na}_{3}$ by the ground state of $\mathrm{Na}$ atom in the high-spin electronic state stabilizes the system to a similar extent as the bond formation energy of the ${ }^{3} \Sigma_{u}^{+} \mathrm{Na}_{2}$ molecule, $0.013 \mathrm{eV}$ (with optimized length 10.493 bohr) in the computational level of $\mathrm{Na}_{3}$ calculations. The triangular $\mathrm{Na}_{3}$ cluster becomes the $D_{3 h}$ regular triangular structure where $r=8.257 \mathrm{bohr}$ and $R=7.151$ bohr by geometry optimization, and the stabilization energy from the ${ }^{2} S \mathrm{Na}+{ }^{3} \Sigma_{u}^{+} \mathrm{Na}_{2}$ system is $0.063 \mathrm{eV}$. The Jahn-Teller distortion is ineffective, similarly as in the triangular $\mathrm{Li}_{3}$ cluster. This stabilization energy is quite larger than that of the colinear $\mathrm{Na}_{3}$ cluster, but it is also less than that of the triangular ${ }^{4} A_{2}^{\prime} \mathrm{Li}_{3}$ cluster. We have also discussed the formation of quintet $\mathrm{Na}_{4}$ cluster from the ${ }^{4} A_{2}^{\prime} \mathrm{Na}_{3}$ cluster and the ${ }^{2} S \mathrm{Na}$ atom. The most stable structure of quintet $\mathrm{Na}_{4}$ cluster is the $T_{d}$ regular tetrahedral structure, where the electronic state is ${ }^{5} A_{1}$, equally as $\mathrm{Li}_{4}$ cluster. The optimized $\mathrm{Na}-\mathrm{Na}$ distance is $7.413 \mathrm{bohr}$ and stabilization energy from the ${ }^{2} \mathrm{~S} \mathrm{Na}+{ }^{4} B_{2} \mathrm{Na}_{3}$ system is $0.302 \mathrm{eV}$. Results of calcula-
TABLE III. Interatomic distances and stabilization energies in high-spin state cluster formation of lithium and sodium at the MRCI level with $6-31 \mathrm{G}^{*}$ basis set.

\begin{tabular}{ccccccc}
\hline \hline & & \multicolumn{3}{c}{$\mathrm{Li}$} & & \multicolumn{2}{c}{$\mathrm{Na}$} \\
\cline { 6 - 7 } \cline { 5 - 6 } & $\begin{array}{c}M= \\
\text { State }\end{array}$ & $\begin{array}{c}\text { M-M distance } \\
\text { (bohr) }\end{array}$ & $\begin{array}{c}\text { Stabilization } \\
\text { energy }(\mathrm{eV})\end{array}$ & $\begin{array}{c}\text { M-M distance } \\
\text { (bohr) }\end{array}$ & $\begin{array}{c}\text { Stabilization } \\
\text { energy (eV) }\end{array}$ \\
\hline $\mathrm{M}_{2}$ & ${ }^{3} \Sigma_{u}^{+}$ & 8.372 & $0.027^{\mathrm{a}}$ & & 10.493 & $0.013^{\mathrm{a}}$ \\
$\mathrm{M}_{3}$ & ${ }^{4} \Sigma_{u}^{+}$ & 7.589 & $0.050^{\mathrm{b}}$ & & 10.192 & $0.016^{\mathrm{b}}$ \\
& ${ }^{4} A_{2}{ }^{\prime}$ & 6.090 & $0.327^{\mathrm{b}}$ & & 8.257 & $0.063^{\mathrm{b}}$ \\
$\mathrm{M}_{4}$ & ${ }^{5} A_{1}$ & 5.822 & $0.773^{\mathrm{c}}$ & & 7.413 & $0.302^{\mathrm{c}}$ \\
\hline \hline
\end{tabular}

${ }^{\text {a Stabilization energy from }}{ }^{2} S \mathrm{M}+{ }^{2} S \mathrm{M}$.

${ }^{\mathrm{b}}$ Stabilization energy from ${ }^{2} S \mathrm{M}+{ }^{3} \Sigma_{u}^{+} \mathrm{M}_{2}$.

${ }^{\mathrm{c}}$ Stabilization energy from ${ }^{2} S \mathrm{M}+{ }^{4} A_{2}^{\prime} \mathrm{M}_{3}$.

tions by using the $6-31 \mathrm{G}^{*}$ basis set are tabulated in Table III. It can be concluded that the stabilization in formation of lithium clusters is much superior to that of sodium clusters.

\section{CONCLUSIONS}

We have carried out ab initio calculations for $\mathrm{Li}_{n}$ and $\mathrm{Na}_{n}$ clusters $(n=2-4)$ for various high-spin electronic states by the MRCI method, and have elucidated the interaction between atoms in terms of the quantum-mechanical energy densities ${ }^{43}$ based on the regional density functional theory. ${ }^{39-49}$ In particular, the electronic kinetic-energy density $n_{T}(\vec{r})$ and the electronic tension density $\vec{\tau}^{S}(\vec{r})$ have been applied to the local electronic nature in the formation of lithium clusters. When the separated two $R_{D} \mathrm{~S}$ in the $\mathrm{Li}_{2}$ molecule touch, it is observed that ratios of occupation on configurations change rapidly; for example, a ratio of the occupation on the excited configurations, which intensify the covalent interaction between Li atoms, starts to increase rapidly in the continuous $R_{D}$ as two $\mathrm{Li}$ atoms approach for the ${ }^{3} \Sigma_{u}^{+}$state of the $\mathrm{Li}_{2}$ molecule. These results are considered as one of the evidences that valence electrons can move around both two Li atoms freely in the meaning of classical mechanics. The shape of $R_{D}$ depends strongly on the electronic state and represents the characteristics of interaction clearly, in particular, for the repulsive interaction like the ${ }^{3} \Pi_{g}$ state of $\mathrm{Li}_{2}$ molecule. The $\vec{\tau}^{S}(\vec{r})$ also gives new images of microscopic electronic stresses.

Furthermore, we have discussed the propagation of $\mathrm{Li}_{n}$ cluster for the high-spin electronic state according to the results of calculations for the quintet states of $\mathrm{Li}_{4}$ cluster system. We have clarified that the most stable structure of $\mathrm{Li}_{3}$ and $\mathrm{Li}_{4}$ cluster systems has the $D_{3 h}$ symmetry and the $T_{d}$ symmetry for the high-spin electronic state, respectively. This result is different from the results for the low-spin electronic state. ${ }^{31-38}$ Particularly, it is important that the most stable $\mathrm{Li}_{4}$ structure is not planar. The stabilization energy due to taking in a $\mathrm{Li}$ atom is raised step by step as the number of atoms in $\mathrm{Li}_{n}$ cluster increases in the initial stage of cluster propagation. It is considered that the most initial stage of cluster propagation would proceed smoothly by spherical.

We have also calculated sodium clusters for the highspin electronic state, and distinguishing characteristics of interaction in lithium clusters from other alkali metals have 
been discussed by comparison between lithium and sodium clusters. In the calculations of $\mathrm{Na}_{2}$ molecule, it is clearly found that the interaction between $\mathrm{Na}$ atoms is weaker than each corresponding electronic state of $\mathrm{Li}_{2}$ molecule. The formation energies of $\mathrm{Na}_{3}$ and $\mathrm{Na}_{4}$ clusters are much smaller than that of the corresponding lithium clusters, respectively. That is, it can be concluded that the stabilization in formation of lithium clusters is quite superior to that of sodium clusters. For $n=2$, the small absolute energy difference between the ${ }^{3} \Sigma_{u}^{+}$state and the ${ }^{3} \Pi_{u}$ state in $\mathrm{Li}_{2}$ molecule would help the excitation to the ${ }^{3} \Pi_{u}$ state as a deep potential well, but it would be hard to take the $\mathrm{Na}_{2}$ molecule in the ${ }^{3} \Pi_{u}$ state because of the large ${ }^{3} \Sigma_{u}^{+}-{ }^{3} \Pi_{u}$ energy difference.

\section{ACKNOWLEDGMENT}

This work was supported by a Grant-in-Aid for Scientific Research from the Ministry of Education, Science and Culture of Japan, for which the authors express their gratitude.

${ }^{1}$ A. Einstein, Sitzungsber. K. Preuss. Akad. Wiss., Phys. Math. K1. 1924, 261.

${ }^{2}$ S. N. Bose, Z. Phys. 26, 178 (1924).

${ }^{3}$ A. Griffin, D. W. Snoke, and S. Stringari, Bose-Einstein Condensation (Cambridge University Press, Cambridge, 1995).

${ }^{4}$ M. H. Anderson, J. R. Ensher, M. R. Matthews, C. E. Wieman, and E. A. Cornell, Science 269, 198 (1995).

${ }^{5}$ K. B. Davis, M.-O. Mewes, M. R. Andrews, N. J. van Druten, D. S. Durfee, D. M. Kurn, and W. Ketterle, Phys. Rev. Lett. 75, 3969 (1995).

${ }^{6}$ C. C. Bradley, C. A. Sackett, J. J. Tollett, and R. G. Hulet, Phys. Rev. Lett. 75, 1687 (1995).

${ }^{7}$ C. C. Bradley, C. A. Sackett, and R. G. Hulet, Phys. Rev. Lett. 78, 985 (1997).

${ }^{8}$ D. G. Fried, T. C. Killian, L. Willmann, D. Landhuis, S. C. Moss, D. Kleppner, and T. J. Greytak, Phys. Rev. Lett. 81, 3811 (1998).

${ }^{9}$ M. R. Andrews, C. G. Townsend, H.-J. Miesner, D. S. Durfee, D. M. Kurn, and W. Ketterle, Science 275, 637 (1997).

${ }^{10}$ J. J. Tollett, C. C. Bradley, C. A. Sackett, and R. G. Hulet, Phys. Rev. A 51, R22 (1995).

${ }^{11}$ M. L. Olson and D. D. Konowalow, Chem. Phys. 21, 393 (1977).

${ }^{12}$ M. L. Olson and D. D. Konowalow, Chem. Phys. 22, 29 (1977).

${ }^{13}$ D. D. Konowalow and M. L. Olson, J. Chem. Phys. 71, 450 (1979).

${ }^{14}$ D. D. Konowalow and J. L. Fish, Chem. Phys. 77, 435 (1983).

${ }^{15}$ D. D. Konowalow and J. L. Fish, Chem. Phys. 84, 463 (1983).

${ }^{16}$ B. Johnsson, B. O. Roos, P. R. Tayler, and P. E. M. Siegbahn, J. Chem. Phys. 74, 4566 (1981).

${ }^{17}$ H. Partridge, C. W. Bauschlicher, Jr., and P. E. M. Siegbahn, Chem. Phys. Lett. 97, 198 (1983).

${ }^{18}$ D. K. Watson, C. J. Cerjan, S. L. Guberman, and P. E. M. Siegbahn, Chem. Phys. Lett. 50, 181 (1977).

${ }^{19}$ G. Das, J. Chem. Phys. 46, 1568 (1967).

${ }^{20}$ B. J. Laurenzi and D. D. Konowalow, Chem. Phys. Lett. 107, 53 (1984).

${ }^{21}$ M. E. Rosenkrantz, R. M. Regan, and D. D. Konowalow, J. Phys. Chem. 89, 2804 (1985).
${ }^{22}$ R. Cote, A. Dalgarno, and M. J. Jamieson, Phys. Rev. A 50, 399 (1994).

${ }^{23}$ J. M. Doyle, B. Friedrich, J. Kim, and D. Patterson, Phys. Rev. A 52, R2515 (1995).

${ }^{24}$ J. T. Bahns, W. C. Stwalley, and P. L. Gould, J. Chem. Phys. 104, 9689 (1996).

${ }^{25}$ Y. B. Band and P. S. Julienne, Phys. Rev. A 51, R4317 (1995).

${ }^{26}$ B. Friedrich and D. Herschbach, Phys. Rev. Lett. 74, 4623 (1995).

${ }^{27}$ K. M. Jones, S. Maleki, L. P. Ratliff, and P. D. Lett, J. Phys. B 30, 289 (1997).

${ }^{28}$ W. T. Zemke and W. C. Stwalley, J. Phys. Chem. 97, 2053 (1993).

${ }^{29}$ E. R. I. Abraham, W. I. McAlexander, C. A. Sackett, and R. G. Hulet, Phys. Rev. Lett. 74, 1315 (1995).

${ }^{30}$ A. J. Moerdijk, W. C. Stwalley, R. G. Hulet, and B. J. Verhaar, Phys. Rev. Lett. 72, 40 (1994).

${ }^{31}$ R. K. Rao, S. N. Khanna, and P. Jena, Phys. Rev. B 36, 953 (1987).

${ }^{32}$ S. Quassowski and K. Hermann, Phys. Rev. B 51, 2457 (1995).

${ }^{33}$ G. Gardet, F. Rogemond, and H. Chermette, Theor. Chim. Acta 91, 249 (1995).

${ }^{34}$ G. Gardet, F. Rogemond, and H. Chermette, J. Chem. Phys. 105, 9933 (1996).

${ }^{35}$ O. Novaro, Can. J. Chem. 74, 825 (1996).

${ }^{36}$ I. G. Kaplan, J. Hernandes-Cobos, I. Ortega-Blake, and O. Novaro, Phys. Rev. A 53, 2493 (1996).

${ }^{37}$ R. O. Jones, A. I. Lichtenstein, and J. Hutter, J. Chem. Phys. 106, 4566 (1997).

${ }^{38}$ R. Rousseau and D. Marx, Phys. Rev. A 56, 617 (1997).

${ }^{39}$ A. Tachibana, Int. J. Quantum Chem., Quantum Chem. Symp. 21, 181 (1987).

${ }^{40}$ A. Tachibana and R. G. Parr, Int. J. Quantum Chem. 41, 527 (1992).

${ }^{41}$ A. Tachibana, Int. J. Quantum Chem. 57, 423 (1996).

${ }^{42}$ A. Tachibana, Theor. Chem. Acc. 102, 188 (1999).

${ }^{43}$ A. Tachibana, J. Chem. Phys. 115, 3497 (2001).

${ }^{44}$ A. Tachibana, K. Nakamura, K. Sakata, and T. Morisaki, Int. J. Quantum Chem. 74, 669 (1999).

${ }^{45}$ A. Tachibana and K. Nakamura, J. Am. Chem. Soc. 117, 3605 (1995).

${ }^{46}$ A. Tachibana, S. Kawauchi, K. Nakamura, and H. Inaba, Int. J. Quantum Chem. 57, 673 (1996)

${ }^{47}$ K. Nakamura, O. Makino, A. Tachibana, and K. Matsumoto, J. Organomet. Chem. 611, 514 (2000).

${ }^{48}$ K. Nakamura, T. Hayashi, A. Tachibana, and K. Matsumoto, J. Cryst. Growth 221, 765 (2000).

${ }^{49} \mathrm{~K}$. Nakamura, A. Tachibana, and K. Matsumoto, in Proceedings of International Workshop on Nitride Semiconductors (The Institute of Pure and Applied Physics, Tokyo, 2000) p. 97.

${ }^{50}$ Molecular Regional DFT program package ver. 1, Tachibana Lab., Kyoto University, Kyoto (in preparation).

${ }^{51}$ P.-O. Widmark, P.-A. Malmqvist, and B. O. Roos, Theor. Chim. Acta 77, 291 (1990).

${ }^{52}$ H.-J. Werner and P. J. Knowles, J. Chem. Phys. 89, 5803 (1988).

${ }^{53}$ P. J. Knowles and H.-J. Werner, Chem. Phys. Lett. 145, 514 (1988).

${ }^{54}$ W. J. Hehre, R. Ditchfield, and J. A. Pople, J. Chem. Phys. 56, 2257 (1972)

${ }^{55}$ P. C. Hariharan and J. A. Pople, Theor. Chim. Acta 28, 213 (1973).

${ }^{56}$ M. S. Gordon, Chem. Phys. Lett. 76, 163 (1980).

${ }^{57}$ M. D. Jones, G. Ortiz, and D. M. Ceperley, Int. J. Quantum Chem. 64, 523 (1997).

${ }^{58}$ P. Schmelcher, Int. J. Quantum Chem. 64, 553 (1997).

${ }^{59}$ K. Runge and J. R. Sabin, Int. J. Quantum Chem. 64, 561 (1997).

${ }^{60}$ R. Jost, Int. J. Quantum Chem. 64, 571 (1997). 